TRANSACTIONS OF THE

AMERICAN MATHEMATICAL SOCIETY

Volume 354, Number 1, Pages 179-203

S 0002-9947(01)02703-9

Article electronically published on May 14, 2001

\title{
POLYTOPAL LINEAR RETRACTIONS
}

\author{
WINFRIED BRUNS AND JOSEPH GUBELADZE
}

\begin{abstract}
We investigate graded retracts of polytopal algebras (essentially the homogeneous rings of affine cones over projective toric varieties) as polytopal analogues of vector spaces. In many cases we show that these retracts are again polytopal algebras and that codimension 1 retractions factor through retractions preserving the semigroup structure. We expect that these results hold in general.

This paper is a part of the project started by the authors in 1999, where we investigate the graded automorphism groups of polytopal algebras. Part of the motivation comes from the observation that there is a reasonable 'polytopal' generalization of linear algebra (and, subsequently, that of algebraic $K$-theory).
\end{abstract}

\section{InTRODUCTION}

The category Vect $_{k}$ of finite-dimensional vector spaces over a field $k$ has a natural extension that we call the polytopal k-linear category $\mathrm{Pol}_{k}$. The objects of $\mathrm{Pol}_{k}$ are the polytopal semigroup algebras (or just polytopal algebras) in the sense of [BGT]. That is, an object $A \in\left|\mathrm{Pol}_{k}\right|$ is (up to graded isomorphism) a standard graded $k$-algebra $k[P]$ associated with a lattice polytope $P \subset \mathbb{R}^{n}$. The morphisms of $\mathrm{Pol}_{k}$ are the homogeneous $k$-algebra homomorphisms. Here, as usual, 'standard graded' means 'graded and generated in degree 1', and a lattice polytope is a finite convex polytope whose vertices are lattice points of the integral lattice $\mathbb{Z}^{n} \subset \mathbb{R}^{n}$.

The generators of $k[P]$ correspond bijectively to the lattice points in $P$, and their relations are the binomials representing the affine dependencies of the lattice points. More precisely, let $\mathrm{L}_{P}$ denote the set of lattice points in $P$. Then $S_{P}$ is the sub-semigroup of $\mathbb{Z}^{n+1}$ generated by

$$
\mathrm{E}_{P}=\left\{(x, 1) \mid x \in \mathrm{L}_{P}\right\} .
$$

and $k[P]=k\left[S_{P}\right]$ is the semigroup algebra of $S_{P}$ with coefficients in $k$ (in BGT. BG1 BG2 we have always used the notation $\left.k\left[S_{P}\right]\right)$. Unless specified otherwise, we will identify the sets $\mathrm{L}_{P}$ and $\mathrm{E}_{P}$.

The category $\mathrm{Pol}_{k}$ contains Vect $k$ as a full subcategory. In fact, we can identify a vector space with the degree 1 component of its symmetric algebra, which, upon the choice of a basis, can be considered as a polynomial ring $k\left[X_{1}, \ldots, X_{n}\right]$. This polynomial ring is isomorphic to the polytopal algebra $k\left[\Delta_{n-1}\right]$ defined by the $(n-1)$-simplex $\Delta_{n-1}$. Vector space homomorphisms extend to homomorphisms of

Received by the editors January 10, 2000 and, in revised form, April 10, 2000.

2000 Mathematics Subject Classification. Primary 13F20, 14M25; Secondary 52 C07.

Key words and phrases. Polytopal algebra, retracts, affine semigroup ring, binomial ideal.

The second author was supported by the Max-Planck-Institut für Mathematik in Bonn and INTAS, Grant 93-2618-Ext. 
symmetric algebras, and thus to homomorphisms of the corresponding polytopal algebras.

Observe that polytopal algebras are essentially the homogeneous coordinate rings of projective normal toric varieties. Actually, one needs the extra condition of very ampleness for the underlying polytope to get such a homogeneous coordinate ring (see [BG1, §5] for details).

Our investigation of polytopal algebras is motivated by two closely related goals: (1) to find the connections between the combinatorial structure of $P$ and the algebraic structure of $k[P]$, and (2) to extend theorems valid in $\operatorname{Vect}_{k}$ to $\operatorname{Pol}_{k}$.

It follows from $[\mathrm{Gu}]$ that an algebra isomorphism of $k[P]$ and $k[Q]$ implies the isomorphism of $P$ and $Q$ as lattice polytopes. This result identifies the objects of the category Pol of lattice polytopes with the objects of $\mathrm{Pol}_{k}$ (up to isomorphism), but there remains the question to which extent the morphisms in $\mathrm{Pol}_{k}$ are determined by those in Pol, namely the $\mathbb{Z}$-affine maps between lattice polytopes. Similarly, one must ask whether certain classes of morphisms in $\mathrm{Pol}_{k}$ can be described in the same way as the corresponding classes in Vect $_{k}$.

As we have shown in BG1, there is a total analogy with the linear situation for the automorphism groups in $\mathrm{Pol}_{k}$ (called polytopal linear groups in [BG1]): they are generated by elementary automorphisms (generalizing elementary matrices), toric automorphisms (generalizing diagonal invertible matrices) and automorphisms of the underlying polytope; moreover, there are normal forms for such representations of arbitrary automorphisms. (In [BG2] this analogy has been extended to automorphisms of so-called polyhedral algebras.)

In the present paper we study retractions in $\mathrm{Pol}_{k}$, that is, idempotent homogeneous endomorphisms of polytopal algebras and their images. Our results support the following two conjectures:

Conjecture A. The images of retractions in $\mathrm{Pol}_{k}$ are polytopal algebras.

Conjecture B. A codimension 1 retraction factors through either a facet projection or an affine lattice retraction of the underlying lattice polytope.

These conjectures generalize the standard facts that every finitely generated vector space has a basis and that an idempotent matrix is conjugate to a sub-unit matrix (ones and zeros on the main diagonal and zeros anywhere else). Conjecture $\mathrm{B}$ must be restricted to codimension 1 since there exist counterexamples for higher codimension.

In the following we say that a retraction $h$ of a polytopal algebra is polytopal if $\operatorname{Im}(h)$ is a polytopal algebra, and codimension 1 retractions satisfying the stronger condition of Conjecture B are called tame (the notion of tameness has a natural generalization to higher codimension). We will provide evidence supporting Conjectures A and B by proving them in a number of special cases (we always assume that the field $k$ is algebraically closed):

(1) If $\operatorname{dim} \operatorname{Im}(h) \leq 2$, then $h$ is polytopal (Theorem 2.2).

(2) Retractions of normal polytopal algebras $C$ that arise from a tensor product decomposition $A \otimes B=C$ of graded $k$-algebras are polytopal; in other words, graded tensor factors of normal polytopal algebras are again polytopal (Theorem 3.11).

(3) If $\operatorname{Im}(h)=k[Q]$ for a lattice subpolytope $Q$ of $P$ such that $Q$ meets the interior of $P$, then $h$ is tame (Theorem[7.2). 
(4) If $\operatorname{Im}(h)=k[Q]$, but the subpolytope $Q$ is contained in the boundary of $P$, then $h$ need not be tame (Examples 5.2 and 5.3 ) in general. However, it is tame if $\operatorname{codim}(h)=1$ (Theorem [7.3).

(5) If $h$ is a codimension 1 retraction such that gr. aut $k(\operatorname{Im}(h))$ contains a torus of dimension $\operatorname{dim} \operatorname{Im}(h)$ whose action can be extended algebraically to $k[P]$, then $h$ is tame (Theorem 7.5).

(6) If $\operatorname{dim}(P) \leq 2$, then all codimension 1 retractions of $k[P]$ are tame (Theorem 8.1).

In Section 6 we discuss the class of segmentonomial ideals, that is, ideals generated by polynomials $f$ whose Newton polytope has dimension $\leq 1$. Section 4 contains a review of the results of [BG1].

Conventions and terminology. The basic facts about affine semigroup rings can be found in [BH, Chap. 6] and [Gi]. For toric varieties we refer the reader to [Fu, Oda]. Newton polytopes are treated in [GKZ].

By $k$ we will always denote a field, and we set $k^{*}=k \backslash\{0\}$. $\mathbb{Z}, \mathbb{Q}, \mathbb{R}$ are the rational, integer and real numbers and $\mathbb{Z}_{+}, \mathbb{Q}_{+}, \mathbb{R}_{+}$are the sub-semigroups of non-negative elements.

An affine semigroup is a finitely generated subsemigroup of a free abelian group. The group of differences of a semigroup $S$ will be denoted by $\operatorname{gp}(S)$. An affine semigroup is positive if its maximal subgroup is trivial. A positive affine semigroup can be embedded into a free commutative semigroup $\mathbb{Z}_{+}^{m}$, thus the semigroup ring $k[S]$ has a (non-unique) grading $k[S]=k \oplus A_{1} \oplus A_{2} \oplus \cdots$ making all nontrivial elements of $S$ homogeneous of positive degree. The uniquely determined minimal generating set of a positive affine semigroup $S$ coincides with its set of indecomposable elements.

In $\mathbb{R} \otimes \operatorname{gp}(S)$ the semigroup $S$ spans the cone $C(S)$.

An affine semigroup $S$ is called homogeneous if $S$ does not have nontrivial invertibles and its irreducible elements are contained in some proper hyperplane of $\mathbb{R} \otimes \operatorname{gp}(S)$. This condition is equivalent to the requirement that the semigroup ring $k[S]$ is a standard graded ring in which $S$ consists of homogeneous elements. Alternatively, there is a lattice polytope $P$ and a subset $X \subset \mathrm{L}_{P}$ such that $k[S]$ is isomorphic to the subalgebra of $k[P]$ generated by $X$.

We put $\operatorname{rank}(S)=\operatorname{rank}(\operatorname{gp}(S))$. An affine semigroup $S$ is called normal if

$$
\forall x \in \operatorname{gp}(S),(c \in \mathbb{N}, c x \in S) \Rightarrow(x \in S) .
$$

The normality of $S$ is equivalent to that of $k[S]$.

The elements of an affine semigroup $S$ will be called terms of $k[S]$, while the elements of type as $\in k[S], a \in k, s \in S$, will be called monomials.

For an affine semigroup $S$ we have the embeddings

$$
S \subset \operatorname{gp}(S) \subset \mathbb{R} \otimes \operatorname{gp}(S) \approx \mathbb{R}^{\operatorname{rank}(S)} .
$$

Therefore, we can speak of the convex hull $\operatorname{conv}(X)$ of a subset $X \subset S$ in $\mathbb{R} \otimes \operatorname{gp}(S)$. For an element $f \in k[S]$ there is a unique canonical representation

$$
f=\sum_{s \in S} a_{s} s, \quad a_{s} \in k .
$$

The polytope

$$
\operatorname{conv}\left(\left\{s \in S \mid a_{s} \neq 0\right\}\right) \subset \mathbb{R} \otimes \operatorname{gp}(S)
$$


is called the Newton polytope of $f$, which we denote by $\mathrm{N}(f)$. We will frequently use the formula

$$
\mathrm{N}(f g)=\mathrm{N}(f)+\mathrm{N}(g)
$$

where ' + ' denotes the Minkowski sum of convex polytopes

$$
P+Q=\{x+y \mid x \in P, y \in Q\} \subset \mathbb{R}^{n}, \quad P \subset \mathbb{R}^{n}, Q \subset \mathbb{R}^{n} .
$$

As remarked already, a lattice polytope $P \subset \mathbb{R}^{n}$ is a finite convex polytope whose vertices belong to the integral lattice $\mathbb{Z}^{n}$. It is called normal if $k[P]$ is normal.

Let $S \subset \mathbb{Z}^{n}$ be an affine semigroup. The semigroup operation in $S$ is written additively when we do linear algebra in $\mathbb{Z}^{n}$, and multiplicatively when $S$ is considered as the set of terms in $k[S]$.

Finally, we adopt the blanket assumption that the field $k$ is algebraically closed, unless specified otherwise.

\section{Retracts of Dimension two}

A retract of a $k$-algebra $A$ is an algebra $B$ such that there exist $k$-homomorphisms $f: B \rightarrow A$ and $g: A \rightarrow B$ with $g \circ f=1_{B}$. This is equivalent to saying that there is an endomorphism $h: A \rightarrow A$ such that $h^{2}=h$ and $\operatorname{Im}(h) \approx B$. We will call such $g$ and $h$ retractions and will frequently make passages between the two equivalent definitions. Moreover, all the retractions considered below are supposed to be graded. For a retraction $h$ as above we put

$$
\operatorname{codim}(h)=\operatorname{dim}(A)-\operatorname{dim}(B) .
$$

The arguments used in the sequel need $k$ to be algebraically closed. We do not know whether the following implication holds for a retraction $h: A \rightarrow A, A \in\left|\operatorname{Pol}_{k}\right|$, over a not necessarily algebraically closed field $k$ :

$$
\bar{k} \otimes h \text { is polytopal (tame) } \Longrightarrow h \text { is polytopal (tame) }
$$

( $\bar{k}$ is the algebraic closure).

That polytopality is in general not an invariant property under scalar extension/restriction is exhibited by the following

Example 2.1. Consider the standard graded $\mathbb{R}$-algebra

$$
A=\mathbb{R}[X, Y, Z] /\left(X^{2}+Y^{2}+Z^{2}\right) .
$$

Then $A$ is a factorial non-polytopal algebra over $\mathbb{R}$ while $\mathbb{C} \otimes A$ is isomorphic to the polytopal algebra $\mathbb{C}\left[2 \Delta_{1}\right]$ defined by a lattice segment $2 \Delta_{1}$ of length 2 .

The factoriality of $A$ is proved in [Fo, $\S 11]$. But the only factorial polytopal algebras (over any field) are polynomial algebras - an easy observation. Hence $A$ is not polytopal because it is singular at the irrelevant maximal ideal. But we have the isomorphism

$$
\alpha: \mathbb{C}\left[U^{2}, U V, V^{2}\right]=\mathbb{C}\left[2 \Delta_{1}\right] \rightarrow \mathbb{C} \otimes A
$$

defined by $U^{2} \mapsto X+i Y, V^{2} \mapsto X-i Y, U V \mapsto i Z$.

Conjecture A holds in Krull dimension $\leq 2$ :

Theorem 2.2. A retract $B$ of a polytopal algebra $A$ is polytopal if $\operatorname{dim} B \leq 2$.

The crucial step in the proof is 
Proposition 2.3. Let $k$ be an algebraically closed field and $A$ a standard graded $k$-algebra of dimension 2. If $A$ is a normal domain and the class group $\mathrm{Cl}(A)$ is finitely generated, then $A$ is isomorphic to $k\left[c \Delta_{1}\right]$ as a graded $k$-algebra for some $c \in \mathbb{N}$ (as usual, $\Delta_{1}$ is the unit segment).

Proof. We have the projectively normal embedding of $\operatorname{Proj}(A)$ given by $A$. Therefore, the projective curve $\operatorname{Proj}(A)$ is normal and thus smooth. Consider the exact sequence

$$
0 \rightarrow \mathbb{Z} \rightarrow \mathrm{Cl}(\operatorname{Proj}(A)) \rightarrow \mathrm{Cl}(A) \rightarrow 0
$$

of Weil divisors arising from viewing $\operatorname{Spec}(A)$ as a cone over $\operatorname{Proj}(A)([\mathrm{Ha}$, Ex. II.6.3(b)]). Since $\mathrm{Cl}(A)$ is finitely generated, so is $\mathrm{Cl}(\operatorname{Proj}(A))$. In particular, the Jacobian $\mathcal{J}(\operatorname{Proj}(A)) \approx \mathrm{Cl}^{0}(\operatorname{Proj}(A))$ is trivial $\left(\mathrm{Cl}^{0}\right.$ denotes degree zero divisor classes). Therefore, the genus of $\operatorname{Proj}(A)$ is 0 , or equivalently $\operatorname{Proj}(A) \approx \mathbb{P}_{k}^{1}$. Using the normality of $A$ once again we get

$$
A \approx \bigoplus_{i=0}^{\infty} H^{0}\left(\mathbb{P}_{k}^{1}, \mathcal{L}^{\otimes i}\right)
$$

for some very ample line bundle $\mathcal{L}$ on $\mathbb{P}_{k}^{1}$. But due to the equality $\operatorname{Pic}\left(\mathbb{P}_{k}^{1}\right)=\mathbb{Z}$ such a line bundle is a positive multiple of $\mathcal{O}(1)$, and hence $A$ is the Veronese subalgebra of the polynomial algebra $k\left[\Delta_{1}\right]$ of some level $c \in \mathbb{N}$.

Proof of Theorem [2.2. In case $\operatorname{dim}(B)=1$ it is easy to see that $B \approx k[X]$.

Consider the case $\operatorname{dim}(B)=2$. We write $A=k[P]$ and denote the retraction $A \rightarrow B \subset A$ by $g$. Consider the set $\left(S_{P} \cap \operatorname{Ker}(g)\right) \subset k[P]$ of monomials. There is a unique face $F \subset P$ such that $\left(S_{P} \cap \operatorname{Ker}(g)\right)=\left(S_{P} \backslash S_{F}\right)$ and $k[P] /\left(S_{P} \cap \operatorname{Ker}(g)\right)$ is naturally isomorphic to $k[F]$. Then $g$ is a composite of the two retractions

$$
k[P] \stackrel{\pi}{\rightarrow} k[F] \stackrel{\rho}{\rightarrow} B
$$

where $\rho$ is the homomorphism induced by $g$. Observe that $\rho$ is in fact a retraction as it is split by $\left.\pi\right|_{B}$.

Therefore from the beginning we can assume that $\left(S_{P} \cap \operatorname{Ker}(g)\right)=0$. In this situation $g$ extends (uniquely) to the normalizations

$$
\overline{k[P]}=k\left[\bar{S}_{P}\right] \stackrel{\bar{g}}{\rightarrow} \bar{B}
$$

This extension is given by

$$
\forall z \in \bar{S}_{P}, \quad \bar{g}(z)=\frac{g(x)}{g(y)}, \quad \text { where } x, y \in S_{P} \text { and } z=\frac{x}{y} .
$$

It is known that the semigroup $S_{n P}$ is normal for all natural numbers $n \geq \operatorname{dim}(P)-1$ $[\mathrm{BGT}$. Therefore, by restricting the retraction (1) to the $n$th Veronese subalgebra for such a number $n$, we get the retraction

$$
k[n P] \stackrel{\bar{g}_{n}}{\longrightarrow} \bar{B}_{(n)} .
$$

Let us show that $\operatorname{Cl}\left(\bar{B}_{(n)}\right)$ is finitely generated for $n \geq \operatorname{dim}(P)-1$. We choose a lattice point $x$ of $S_{n P}$ that is in the interior of the cone $C\left(S_{P}\right)$. By localization (2) gives rise to the retraction

$$
\left(x \bar{g}_{n}(x)\right)^{-1} k[n P] \rightarrow\left(\bar{g}_{n}(x)\right)^{-1} \bar{B}_{(n)} .
$$

Since $\left(x \bar{g}_{n}(x)\right)^{-1} k[n P]$ is a localization of the Laurent polynomial ring $x^{-1} k[n P]$ $=k\left[\operatorname{gp}\left(S_{n P}\right)\right]$, it is a factorial ring. Then its retract $\left(\bar{g}_{n}(x)\right)^{-1} \bar{B}_{(n)}$ is factorial as 
well (for example, see $\overline{\mathrm{Cos}}$ ). By Nagata's theorem $[\mathrm{Fo}] \mathrm{Cl}\left(\bar{B}_{(n)}\right)$ is generated by the classes of the height 1 prime ideals of $\bar{B}_{(n)}$ containing $\bar{g}_{n}(x)$ - a finite set.

It is also clear from (2) that $\bar{B}_{(n)}$ is generated in degree 1 . Consequently, by Proposition 2.3 for each $n \geq \operatorname{dim}(P)-1$ there is a natural number $c_{n}$ and an isomorphism

$$
\varphi_{n}: \bar{B}_{(n)} \rightarrow k\left[c_{n} \Delta_{1}\right] .
$$

We now fix such a number $n$. Restricting $\varphi_{n}$ and $\varphi_{n+1}$ to the iterated Veronese subalgebra $\bar{B}_{(n(n+1))}=\left(\bar{B}_{(n)}\right)_{(n+1)}=\left(\bar{B}_{(n+1)}\right)_{(n)}$ we obtain two isomorphisms of $\bar{B}_{(n(n+1))}$ with $k\left[c_{n(n+1)} \Delta_{1}\right]$. It follows that there exists $c \in \mathbb{N}$ with $c_{n}=c n$ and $c_{n+1}=c(n+1)$, and furthermore the restrictions of $\varphi_{n}$ and $\varphi_{n+1}$ differ by an automorphism of $k\left[c_{n(n+1)} \Delta_{1}\right]$. However, each automorphism of $k\left[c_{n(n+1)} \Delta_{1}\right]$ can be lifted to an automorphism of $k\left[\Delta_{1}\right]$, and then restricted to all Veronese subrings of $k\left[\Delta_{1}\right]$. (This follows from the main result of [BG1].) Therefore, we can assume that the restrictions of $\varphi_{n}$ and $\varphi_{n+1}$ coincide. Then they define an isomorphism of the subalgebra $V$ of $\bar{B}$ generated by the elements in degree $n$ and $n+1$ to the corresponding subalgebra of $k\left[\Delta_{1}\right]$; see Lemma 2.4 below. Taking normalizations yields an isomorphism $\bar{B} \approx k\left[c \Delta_{1}\right]$. But then $B=k\left[c \Delta_{1}\right]$ as well, because $\bar{B}$ and $B$ coincide in degree 1 (being retracts of algebras with this property).

Lemma 2.4. Let $A$ and $B$ be $\mathbb{Z}$-graded rings. Suppose that $B$ is reduced. If the homogeneous homomorphisms $\varphi: A_{(n)} \rightarrow B_{(n)}$ and $\psi: A_{(n+1)} \rightarrow B_{(n+1)}$ coincide on $A_{(n(n+1))}$, then they have a common extension to a homogeneous homomorphism $\chi: V \rightarrow B$, where $V$ is the subalgebra of $A$ generated by $A_{(n)}$ and $A_{(n+1)}$. If, in addition, $A$ is reduced and $\varphi$ and $\psi$ are injective, then $\chi$ is also injective.

Proof. One checks easily that one only needs to verify the following: if $u v=u^{\prime} v^{\prime}$ for homogeneous elements $u, u^{\prime} \in A_{(n)}, v, v^{\prime} \in A_{(n+1)}$, then $\varphi(u) \psi(v)=\varphi\left(u^{\prime}\right) \psi\left(v^{\prime}\right)$. As $B$ is reduced, it is enough that $\left(\varphi(u) \psi(v)-\varphi\left(u^{\prime}\right) \psi\left(v^{\prime}\right)\right)^{n(n+1)}=0$. Since

$$
u^{p}\left(u^{\prime}\right)^{n(n+1)-p}, v^{p}\left(v^{\prime}\right)^{n(n+1)-p} \in A_{(n(n+1))}, \quad p \in[0, n(n+1)],
$$

this follows immediately from the hypothesis that $\varphi$ and $\psi$ coincide on $A_{(n(n+1))}$.

If $A$ is reduced, then every non-zero homogeneous ideal in $A$ intersects $A_{(n(n+1))}$ non-trivially, and this implies the second assertion.

\section{TENSOR FACTORS}

In this section we examine a special case of conjecture A. Suppose

$$
A=k \oplus A_{1} \oplus A_{2} \oplus \cdots \text { and } B=k \oplus B_{1} \oplus B_{2} \oplus \cdots
$$

are (standard) graded. Then, as usual, their tensor product (over $k$ ) is the (standard) graded algebra

$$
A \otimes_{k} B=k \oplus C_{1} \oplus C_{2} \oplus \cdots, \quad C_{h}=\bigoplus_{i+j=h}\left(A_{i} \otimes_{k} B_{j}\right) .
$$

Clearly, $A$ is a graded retract of $A \otimes B$; one just considers the retraction

$$
A \otimes B \stackrel{1_{A} \otimes \pi_{B}}{\longrightarrow} A \otimes k=A,
$$

where $\pi_{B}: B \rightarrow k$ is the augmentation with $\operatorname{Ker}\left(\pi_{B}\right)$ the irrelevant maximal ideal. 
In the special case of polytopal algebras $A=k[P]$ and $B=k[Q]$ we have the following easily verified formula

$$
k[P] \otimes k[Q] \approx k[\operatorname{join}(P, Q)] .
$$

We recall from [BG2] that a lattice polytope $R$ is a join of two lattice polytopes $P$ and $Q$ if $R$ has two faces isomorphic to $P$ and $Q$ as lattice polytopes, the affine hulls of these faces do not intersect each other in the affine hull of $R$, and $\mathrm{L}_{R}=\mathrm{L}_{P} \cup \mathrm{L}_{Q}$. Clearly, in this situation $R$ is the convex hull of these faces. (Note that $R$ is unique up to an isomorphism of lattice polytopes.)

Theorem 3.1. If $A$ and $B$ are two standard graded $k$-algebras such that $A \otimes B \approx$ $k[P]$ for some normal lattice polytope $P$, then both $A$ and $B$ are polytopal.

In particular, we obtain the splitting off property of polytopal extensions,

$$
(A \otimes k[Q] \approx k[P]) \Rightarrow(A \approx k[R] \text { for some } R)
$$

if $P$ is a normal lattice polytope.

An essential step in the proof of Theorem 3.1 is the special case when one of the factors collapses into the cone over a point, that is a polynomial ring in one variable.

Proposition 3.2. Let $A$ be as in Theorem 3.1 and $P$ be any lattice polytope. If $A[X] \approx k[P]$ as graded algebras ( $X$ a variable), then there is a polytope $R$ for which $A \approx k[R]$.

Remark 3.3. Let us call an ideal $I$ binomial if it is generated by monomials $\alpha T$ and binomials $T-\beta T^{\prime}$ where $\alpha, \beta \in k^{*}$ and $T, T^{\prime}$ denote terms. The arguments in the proof of Proposition [3.2, presented below, yield the following more general result: for a not necessarily algebraically closed field $k$ and an ideal $I \subset k\left[X_{1}, \ldots, X_{n}\right]$ the ideal

$$
I k\left[X_{1}, \ldots, X_{n}, X\right] \subset k\left[X_{1}, \ldots, X_{n}, X\right]
$$

can be made a binomial ideal by a linear automorphism of $k\left[X_{1}, \ldots, X_{n}, X\right]$ if and only if this is possible for $I$ in $k\left[X_{1}, \ldots, X_{n}\right]$.

Several times we will use the following theorem [ES, 2.6] characterizing binomial prime ideals in affine semigroup rings over algebraically closed fields. (In [ES] the theorem is given only for polynomial rings, but the generalization is immediate.)

Theorem 3.4. Let $k$ be an algebraically closed field. A binomial ideal I in an affine semigroup ring $k[S]$ is prime if and only if the residue class ring $k[S] / I$ contains a (multiplicative) affine semigroup $S^{\prime}$ such that $k[S] / I=k\left[S^{\prime}\right]$ and, moreover, the natural epimorphism $k[S] \rightarrow k\left[S^{\prime}\right]$ maps the monomials in $k[S]$ to those in $k\left[S^{\prime}\right]$.

Proof of Proposition 3.2. Since polytopal algebras are precisely the standard graded affine semigroup rings coinciding with their normalizations in degree 1 [BGT], we only need to show that $A$ is a semigroup ring.

Theorem 3.4 reduces the proposition to the assertion on binomial ideals $I$ discussed in Remark 3.3 where the ideal $I$ to be considered is the kernel of a surjection $k\left[X_{1}, \ldots, X_{n}\right] \rightarrow A$.

We set $X_{n+1}=X$. By hypothesis there exists a linear transformation $\Psi$,

$$
\Psi\left(X_{i}\right)=t_{i 1} X_{1}+\cdots+t_{i n} X_{n}+t_{i n+1} X_{n+1}, \quad i \in[1, n+1], \quad t_{i j} \in k,
$$


such that $\Psi\left(I k\left[X_{1}, \ldots, X_{n+1}\right]\right)$ is binomial. For all $j \in[1, n]$ we consider the surjection

$$
\pi_{j}: k\left[X_{1}, \ldots, X_{n+1}\right] \rightarrow k\left[X_{1}, \ldots, X_{n}\right], \quad \pi_{j}\left(X_{i}\right)=X_{i}, \pi_{j}\left(X_{n+1}\right)=X_{j} .
$$

We define the linear endomorphisms $\varepsilon_{0}$ and $\varepsilon_{j}, j \in[1, n]$ of $k\left[X_{1}, \ldots, X_{n}\right]$ by

$$
\begin{aligned}
& \varepsilon_{0}\left(X_{i}\right)=t_{i 1} X_{1}+\cdots+t_{i n} X_{n}, \quad i \in[1, n], \\
& \varepsilon_{j}\left(X_{i}\right)=t_{i 1} X_{1}+\cdots+t_{i n} X_{n}+t_{i n+1} X_{j}, \quad i \in[1, n] .
\end{aligned}
$$

There exists $j$ for which $\varepsilon_{j}$ is an automorphism. In fact, let $T$ denote the $(n+1) \times(n+1)$ matrix $\left(t_{i j}\right)$. If $\varepsilon_{0}$ is not an automorphism, then the $n \times n$ submatrix $T^{\prime}$ of $T$ consisting of the intersection of the first $n$ rows with the first $n$ columns of $T$ has rank $n-1$ and the larger submatrix $T^{\prime \prime}$ consisting of the first $n$ rows has $\operatorname{rank} n$ (otherwise $\operatorname{rank} T<n+1$ ). There exists $j \in[1, n]$ such that the $j$ th column of $T^{\prime}$ lies in the vector space $U$ spanned by the remaining $n-1$ columns of $T^{\prime}$, but the $(n+1)$ th column of $T^{\prime \prime}$ does not belong to $U$. If we replace the $j$ th column of $T^{\prime}$ by its sum with $(n+1)$ th column of $T^{\prime \prime}$, we obtain an $n \times n$ matrix of rank $n$, and this matrix defines the automorphism $\varepsilon_{j}$.

There exists a unique epimorphism $\nu: k\left[X_{1}, \ldots, X_{n}, X_{n+1}\right] \rightarrow k\left[X_{1}, \ldots, X_{n}\right]$ making the diagram

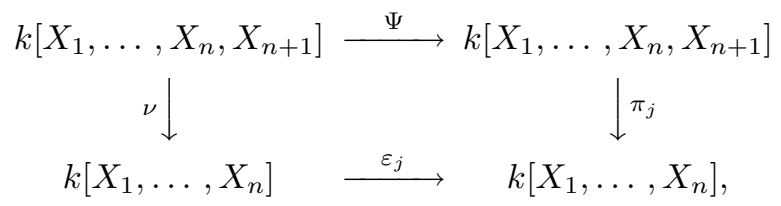

commutative. Since $\nu\left(X_{i}\right)=X_{i}$ for $i \in[1, n]$, we have $\nu\left(I k\left[X_{1}, \ldots, X_{n}, X_{n+1}\right]\right)=$ $I$, and $\pi_{j}$ maps binomial ideals to binomial ideals.

Two more auxiliary facts:

Lemma 3.5. Let $S$ be a positive affine semigroup. Then the graded ring

$$
\operatorname{gr}_{\mathfrak{m}}(k[S])=\operatorname{gr}_{\mathfrak{m}}\left(k[S]_{\mathfrak{m}}\right)
$$

is a domain if and only if $S$ is homogeneous.

Proof. If $S$ is not homogeneous, then among the relations between the irreducible elements of $S$ there occurs an equation

$$
s_{1}^{a_{1}} \cdots s_{r}^{a_{r}}=s_{1}^{b_{1}} \cdots s_{r}^{b_{r}}, \quad a_{1}+\cdots+a_{r}<b_{1}+\cdots+b_{r},
$$

for some $a_{i}, b_{i} \geq 0$. But then ${\overline{s_{1}}}^{a_{1}} \cdots{\overline{s_{r}}}^{a_{r}}=0$ in $\operatorname{gr}_{\mathfrak{m}}(k[S])$, where $\bar{s}_{i}$ denotes the residue class of $s_{i}$ in $\mathfrak{m} / \mathfrak{m}^{2}$. Since these are nonzero classes, we get a contradiction.

The other implication is clear.

Lemma 3.6. Let $S$ be a normal positive affine semigroup. For an edge $E$ of the polyhedral cone $C(S)$ spanned by $S$ in $\mathbb{R} \otimes \operatorname{gp}(S)$ let $x_{E}$ be the generator of the subsemigroup $E \cap S \subset S$ (isomorphic to $\mathbb{Z}_{+}$) and let $x_{E}^{-1} S$ denote the sub-semigroup of $\operatorname{gp}(S)$ generated by $S \cup\left\{x_{E}^{-1}, x_{E}^{-2}, \ldots\right\}$. Then

$$
x_{E}^{-1} S \approx \mathbb{Z} \times S^{\prime}
$$

for some normal positive affine semigroup $S^{\prime}$.

For example, see [Gu. §2]. 
Proof of Theorem [3.1, As $A$ and $B$ are retracts of $k[P]$, they have to be normal domains.

We denote by $X$ and $Y$ the affine normal toric varieties corresponding to $A$ and $B$. The two irrelevant maximal ideals will be denoted by $\mathfrak{m}_{A}$ and $\mathfrak{m}_{B}$ respectively.

Let $y \in Y$ be any smooth point. Then for the local ring of the point $\left(\mathfrak{m}_{A}, y\right) \in$ $X \times Y$ we have

$$
\operatorname{gr}\left(\mathcal{O}_{\left(\mathfrak{m}_{A}, y\right)}\right) \approx A\left[Y_{1}, \ldots, Y_{\operatorname{dim}(B)}\right] .
$$

as graded algebras (the $Y_{i}$ are indeterminates over $A$ ).

On the other hand, identifying $X \times Y$ with the variety $\operatorname{maxSpec}(k[P])$ we see that there is a maximal ideal $\mathfrak{n} \subset k[P]$ such that

$$
\operatorname{gr}\left(\mathcal{O}_{\left(\mathfrak{m}_{A}, y\right)}\right) \approx \operatorname{gr}_{\mathfrak{n}}\left(k[P]_{\mathfrak{n}}\right) .
$$

By an iterated use of Lemma [3.6] we get

$$
k[P]_{\mathfrak{n}} \approx\left(k[S]\left[T_{1}, T_{1}^{-1}, \ldots, T_{s}, T_{s}^{-1}\right]\right)_{\mathfrak{r}}
$$

for some positive normal affine semigroup $S$, an integer $s \geq 0$, and a maximal ideal $\mathfrak{r} \subset k[S]\left[T_{1}, T_{1}^{-1}, \ldots, T_{s}, T_{s}^{-1}\right]$, containing $S \backslash\{1\}$. Since $k$ is algebraically closed, there are elements $a_{1}, \ldots, a_{s} \in k^{*}$ such that $\mathfrak{r}$ is generated by the maximal monomial ideal $\mathfrak{r}_{0} \subset k[S]$ and $\left\{T_{1}-a_{1}, \ldots, T_{s}-a_{s}\right\}$. Therefore, one has a natural graded isomorphism

$$
\operatorname{gr}_{\mathfrak{n}}\left(k\left[\bar{S}_{P}\right]_{\mathfrak{n}}\right) \approx\left(\operatorname{gr}_{\mathfrak{r}_{0}}\left(k[S]_{\mathfrak{r}_{0}}\right)\right)\left[\tau_{1}, \ldots, \tau_{s}\right]
$$

for $\tau_{i}=T_{i}-a_{i}$.

Consequently,

$$
A\left[Y_{1}, \ldots, Y_{\operatorname{dim}(B)}\right] \approx\left(\operatorname{gr}_{\mathfrak{r}_{0}}\left(k[S]_{\mathfrak{r}_{0}}\right)\right)\left[\tau_{1}, \ldots, \tau_{s}\right]
$$

as graded algebras. By Lemma $3.5 S$ is a homogeneous affine semigroup. Then, clearly, $S \approx S_{Q}$ for some normal polytope $Q$ and we get the graded isomorphism

$$
A\left[Y_{1}, \ldots, Y_{\operatorname{dim}(B)}\right] \approx k[Q]\left[\tau_{1}, \ldots, \tau_{s}\right] \approx k\left[\operatorname{join}\left(Q, \Delta_{s-1}\right]\right]
$$

$\left(\Delta_{s-1}\right.$ denotes the $(s-1)$-unit simplex). By an iterated use of Proposition 3.2 we can split off the polynomial extension on the left. That is, $A$ is polytopal. By symmetry, $B$ is also polytopal.

Remark 3.7. As a consequence of 3.2 one can show that any retract of a polytopal algebra defined by a single equation is polytopal. In fact, suppose that $k[P]=k\left[X_{1}, \ldots, X_{n}\right] /(b)$ where $b$ is a binomial of degree $d>0$ and denote the natural epimorphism from $k\left[X_{1}, \ldots, X_{n}\right]$ onto $k[P]$ by $\pi$. Let $h$ be a retraction of $k[P], y_{1}, \ldots, y_{m}$ a $k$-basis of $\operatorname{Im}(h)_{1}$ and $z_{1}, \ldots, z_{p}$ a $k$-basis of $\operatorname{Ker}(h)_{1}$. Let $g: k\left[Y_{1}, \ldots, Y_{m}\right] \rightarrow \operatorname{Im}(h)$ be given by $g\left(Y_{i}\right)=y_{i}$, and let $\gamma$ be the (unique) epimorphism $k\left[X_{1}, \ldots, X_{n}\right] \rightarrow k\left[Y_{1}, \ldots, Y_{m}\right]$ with $g \circ \gamma=h \circ \pi$. Then $\operatorname{Ker}(g)$ is generated by $\pi(b)$. If $\pi(b)=0$, then $\operatorname{Im}(h)$ is a polynomial ring. Otherwise, $b^{\prime}=\pi(b)$ is of degree $d$. Now we extend $g$ to the epimorphism $g^{\prime}:\left(k\left[Y_{1}, \ldots, Y_{m}\right] /\left(b^{\prime}\right)\right)\left[Z_{1}, \ldots, Z_{p}\right] \rightarrow k[P]$ with $g^{\prime}\left(Z_{i}\right)=z_{i}$. Comparing Hilbert functions we see that $g^{\prime}$ is an isomorphism, and Proposition 3.2 shows that $\operatorname{Im}(h) \approx k\left[Y_{1}, \ldots, Y_{m}\right] /\left(b^{\prime}\right)$ is polytopal. 


\section{Polytopal Linear GROUPS}

Here we survey the relevant part of [BG1] that will be used in the sequel. Throughout this section $k$ is a general (not necessarily algebraically closed) field.

For a lattice polytope $P$ the group $\Gamma_{k}(P)=\operatorname{gr}$. aut $(k[P])$ is a linear $k$-group in a natural way. It coincides with $\mathrm{GL}_{n}(k)$ in the case $P$ is the unit $(n-1)$-simplex $\Delta_{n-1}$. The groups $\Gamma_{k}(P)$ are called polytopal linear groups in BG1].

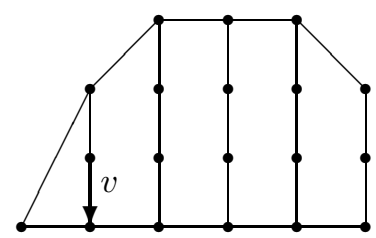

Figure 1. A column structure

An element $v \in \mathbb{Z}^{d}, v \neq 0$, is a column vector (for $P$ ) if there is a facet $F \subset P$ such that $x+v \in P$ for every lattice point $x \in P \backslash F$. The facet $F$ is called the base facet of $v$. The set of column vectors of $P$ is denoted by $\operatorname{Col}(P)$. A pair $(P, v)$, $v \in \operatorname{Col}(P)$, is called a column structure. Let $(P, v)$ be a column structure and $P_{v} \subset P$ be the base facet for $v \in \operatorname{Col}(P)$. Then for each element $x \in S_{P}$ we set ht $_{v}(x)=m$ where $m$ is the largest non-negative integer for which $x+m v \in S_{P}$. Thus ht ${ }_{v}(x)$ is the 'height' of $x$ above the facet of the cone $C\left(S_{P}\right)$ corresponding to $P_{v}$ in direction $-v$. It is an easy observation that $x+\mathrm{ht}_{v}(x) \cdot v \in S_{P_{v}} \subset S_{P}$ for any $x \in S_{P}$. (See Figure1.)

Let $(P, v)$ be a column structure and $\lambda \in k$. We identify the vector $v$, representing the difference of two lattice points in $P$, with the degree 0 element $(v, 0) \in \operatorname{gp}\left(S_{P}\right) \subset$ $k\left[\operatorname{gp}\left(S_{P}\right)\right]$. Then the assignment

$$
x \mapsto(1+\lambda v)^{\mathrm{ht}_{v}(x)} x .
$$

gives rise to a graded $k$-algebra automorphism $e_{v}^{\lambda}$ of $k[P]$. Observe that $e_{v}^{\lambda}$ becomes an elementary matrix in the special case when $P=\Delta_{n-1}$, after the identifications $k\left[\Delta_{n-1}\right]=k\left[X_{1}, \ldots, X_{n}\right]$ and $\Gamma_{k}(P)=\mathrm{GL}_{n}(k)$. Accordingly, $e_{v}^{\lambda}$ is called an elementary automorphism.

In the following $\mathbb{A}_{k}^{s}$ denotes the additive group of the $s$-dimensional affine space.

Proposition 4.1. Let $v_{1}, \ldots, v_{s}$ be pairwise different column vectors for $P$ with the same base facet $F=P_{v_{i}}, i=1, \ldots, s$.

(a) The mapping

$$
\varphi: \mathbb{A}_{k}^{s} \rightarrow \Gamma_{k}(P), \quad\left(\lambda_{1}, \ldots, \lambda_{s}\right) \mapsto e_{v_{1}}^{\lambda_{1}} \circ \cdots \circ e_{v_{s}}^{\lambda_{s}},
$$

is an embedding of algebraic groups. In particular, $e_{v_{i}}^{\lambda_{i}}$ and $e_{v_{j}}^{\lambda_{j}}$ commute for all $i, j \in\{1, \ldots, s\}$ and $\left(e_{v_{1}}^{\lambda_{1}} \circ \cdots \circ e_{v_{s}}^{\lambda_{s}}\right)^{-1}=e_{v_{1}}^{-\lambda_{1}} \circ \cdots \circ e_{v_{s}}^{-\lambda_{s}}$.

(b) For $x \in \mathrm{L}_{P}$ with $\mathrm{ht}_{v_{1}}(x)=1$ one has

$$
e_{v_{1}}^{\lambda_{1}} \circ \cdots \circ e_{v_{s}}^{\lambda_{s}}(x)=\left(1+\lambda_{1} v_{1}+\cdots+\lambda_{s} v_{s}\right) x .
$$

The image of the embedding $\varphi$ given by Lemma 4.1 is denoted by $\mathbb{A}(F)$. Of course, $\mathbb{A}(F)$ may consist only of the identity map of $k[P]$, namely if there is no column vector with base facet $F$. 
Put $n=\operatorname{dim}(P)+1$. The $n$-torus $\mathbb{T}_{n}=\left(k^{*}\right)^{n}$ acts naturally on $k[P]$ by restriction of its action on $k\left[\mathrm{gp}\left(S_{P}\right)\right]$ that is given by

$$
\left(\xi_{1}, \ldots, \xi_{n+1}\right)\left(e_{i}\right)=\xi_{i} e_{i}, \quad i \in[1, n] .
$$

Here $e_{i}$ is the $i$ th element of a fixed basis of $\operatorname{gp}\left(S_{P}\right)=\mathbb{Z}^{n}$. This gives rise to an algebraic embedding $\mathbb{T}_{n} \subset \Gamma_{k}(P)$, whose image we denote by $\mathbb{T}_{k}(P)$. It consists precisely of those automorphisms of $k[P]$ which multiply each monomial by a scalar from $k^{*}$.

The (finite) automorphism group $\Sigma(P)$ of the semigroup $S_{P}$ is also a subgroup of $\Gamma_{k}(P)$.

Theorem 4.2. Let $P$ be a convex lattice $n$-polytope and $k$ a field. Every element $\gamma \in \Gamma_{k}(P)$ has a (not uniquely determined) presentation

$$
\gamma=\alpha_{1} \circ \alpha_{2} \circ \cdots \circ \alpha_{r} \circ \tau \circ \sigma,
$$

where $\sigma \in \Sigma(P), \tau \in \mathbb{T}_{k}(P)$, and $\alpha_{i} \in \mathbb{A}\left(F_{i}\right)$ such that the facets $F_{i}$ are pairwise different and $\# \mathrm{~L}_{F_{i}} \leq \# \mathrm{~L}_{F_{i+1}}, i \in[1, r-1]$.

For any positive affine semigroup $S$ and any grading

$$
k[S]=k \oplus A_{1} \oplus A_{2} \oplus \cdots,
$$

making all non-unit monomials homogeneous of positive degree, the automorphisms of $k[S]$ that multiply monomials by scalars from $k^{*}$ constitute a closed $\operatorname{rank}(S)$-torus

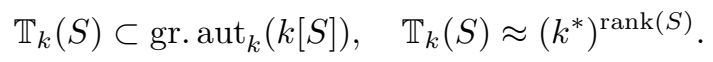

This torus is naturally identified with the set of $k$-rational points of the open subscheme

$$
\operatorname{Spec}(k[\operatorname{gp}(S)]) \subset \operatorname{Spec}(k[S]) .
$$

and is called the embedded torus. In case $k$ is infinite, the embedded torus is maximal (by literally the same arguments as at the end of $\S 3$ in [BG1]).

\section{The Structure of Retractions}

Now we first consider Conjecture B in detail and then observe that it does not admit a direct extension to codimension $\geq 2$.

Let $P \subset \mathbb{R}^{n}$ be a lattice polytope of dimension $n$ and $F \subset P$ a face. Then there is a uniquely determined retraction

$$
\pi_{F}: k[P] \rightarrow k[F], \quad \pi_{F}(x)=0 \quad \text { for } x \in \mathrm{L}_{P} \backslash F .
$$

Retractions of this type will be called face retractions and facet retractions if $F$ is a facet or, equivalently, $\operatorname{codim}\left(\pi_{F}\right)=1$.

Now suppose there are an affine subspace $H \subset \mathbb{R}^{n}$ and a vector subspace $W \subset \mathbb{R}^{n}$ with $\operatorname{dim} W+\operatorname{dim} H=n$, such that

$$
\mathrm{L}_{P} \subset \bigcup_{x \in \mathrm{L}_{P} \cap H}(x+W) .
$$

(Observe that $\operatorname{dim}(H \cap P)=\operatorname{dim} H$.) The triple $(P, H, W)$ is called a lattice fibration of codimension $c=\operatorname{dim} W$, whose base polytope is $P \cap H$; its fibers are the maximal lattice subpolytopes of $(x+W) \cap P, x \in \mathrm{L}_{P} \cap H$ (the fibers may have smaller dimension than $W)$. $P$ itself serves as a total polytope of the fibration. If $W=\mathbb{R} w$ is a line, then we call the fibration segmental and write $(P, H, w)$ for 


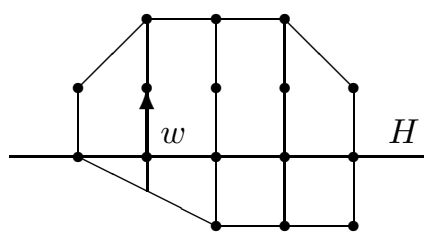

Figure 2. A lattice segmental fibration

it. Note that the column structures introduced in Section 4 give rise to lattice segmental fibrations in a natural way.

For a lattice fibration $(P, H, W)$ let $L \subset \mathbb{Z}^{n}$ denote the subgroup spanned by $\mathrm{L}_{P}$, and let $H_{0}$ be the translate of $H$ through the origin. Then one has the direct sum decomposition

$$
L=(L \cap W) \oplus\left(L \cap H_{0}\right) .
$$

(See Figure 2) Equivalently,

$$
\operatorname{gp}\left(S_{P}\right)=L \oplus \mathbb{Z}=\left(\operatorname{gp}\left(S_{P}\right) \cap W_{1}\right) \oplus \operatorname{gp}\left(S_{P \cap H_{1}}\right) .
$$

where $W_{1}$ is the image of $W$ under the embedding $\mathbb{R}^{n} \rightarrow \mathbb{R}^{n+1}, w \mapsto(w, 0)$, and $H_{1}$ is the vector subspace of $\mathbb{R}^{n+1}$ generated by all the vectors $(h, 1), h \in H$.

For a fibration $(P, H, W)$ one has the naturally associated retraction

$$
\rho_{(P, H, W)}: k[P] \rightarrow k[P \cap H] ;
$$

it maps $\mathrm{L}_{P}$ to $\mathrm{L}_{P \cap H}$ so that fibers are contracted to their intersection points with the base polytope $P \cap H$.

Clearly, if $f: k[P] \rightarrow k[P]$ is a retraction, then for any graded automorphism $\alpha$ of $k[P]$ the composite map $f^{\alpha}=\alpha \circ f \circ \alpha^{-1}$ is again a retraction and $\operatorname{Im}\left(f^{\alpha}\right)=$ $\alpha(\operatorname{Im}(f))$ and $\operatorname{Ker}\left(f^{\alpha}\right)=\alpha(\operatorname{Ker}(f))$. Now the exact formulation of Conjecture B is as follows.

Conjecture B. For a codimension 1 retraction $f: k[P] \rightarrow k[P]$ there is $\alpha \in \Gamma_{k}(P)$ such that $f^{\alpha}=\iota \circ g$ for a retraction $g$ of type either $\pi_{F}$ or $\rho_{(P, H, w)}$ and $\iota: \operatorname{Im}(g) \rightarrow$ $k[P]$ a graded k-algebra embedding.

In other words, this conjecture claims that any codimension 1 retraction can be 'modified' by an automorphism so that the corrected retraction factors through a retraction preserving the monomial structure.

The explicit description of the embeddings $\iota$ is not addressed in this paper.

A necessary condition for Conjecture $\mathrm{B}$ is that any codimension 1 retraction $f$ can be modified by a graded automorphism $\alpha$ so that $f^{\alpha}$ has either a homogeneous binomial of degree 1 or a monomial of degree 1 in its kernel. A weaker condition is that $\operatorname{Ker}\left(f^{\alpha}\right)$ contains a homogeneous binomial of degree $\geq 1$ (evidently this holds if there is a monomial in $\left.\operatorname{Ker}\left(f^{\alpha}\right)\right)$.

We remark that even an example of just an endomorphism in $\mathrm{Pol}_{k}$, such that $\operatorname{Ker}\left(f^{\alpha}\right)$ contains no (homogeneous) binomial for any $\alpha$, is not readily found. However, such exists, even in the class of codimension 2 retractions.

The examples below are constructed from joins of polytopes. The following lemma enables us to describe $\Gamma_{k}(R)$ for $R=\operatorname{join}(P, Q)$ under a mild assumption on $P$ and $Q$. We identify them with the corresponding faces of $R$. 
Lemma 5.1. Let $P$ and $Q$ be lattice polytopes, both having interior lattice points. Then $\operatorname{Col}(\operatorname{join}(P, Q))=\operatorname{Col}(P) \cup \operatorname{Col}(Q)$.

Proof. That each of the column vectors of the polytopes serves as a column vector for join $(P, Q)$ is clear.

Now let $v \in \operatorname{Col}(\operatorname{join}(P, Q))$. If $v$ is parallel to either $P$ or $Q$ then either $v \in$ $\operatorname{Col}(P)$ or $v \in \operatorname{Col}(Q)$ since $\mathrm{L}_{\text {join }(P, Q)}=\mathrm{L}_{P} \cup \mathrm{L}_{Q}$. So without loss of generality we can assume that $v$ is parallel neither to $P$ nor to $Q$. Since $P$ and $Q$ span join $(P, Q)$ they cannot be contained simultaneously in the base facet of $v$. But then either $p+v \in \operatorname{join}(P, Q)$ or $q+v \in \operatorname{join}(P, Q)$ for suitable vertices $p \in P$ and $q \in Q$. We get a contradiction because one of the points $r+v$ or $s+v$ is outside join $(P, Q)$ for interior lattice points $r \in P, s \in Q$.

Example 5.2. Let $Q$ be the lattice triangle spanned by $(0,-1),(-1,0)$, and $(1,1)$. Then $Q$ contains only one more lattice point, namely $(0,0)$. Identifying $U$ with $(0,0), V$ with $(0,-1)$, and $W$ with $(-1,0)$, we see that the polynomial ring $k[U, V, W]$ can be embedded into $k[Q]$ such that the indeterminates correspond to lattice points. Moreover, $k\left[\mathrm{gp}\left(S_{Q}\right)\right]$ is then just the Laurent polynomial ring $k\left[\mathbb{Z}^{3}\right]=k\left[U^{ \pm 1}, V^{ \pm 1}, W^{ \pm 1}\right]$.

Let $h^{\prime}: k[X, Y] \rightarrow k[U, V, W]$ be defined by $h^{\prime}(X)=U+V, h^{\prime}(Y)=U+W$. Then $h^{\prime}$ induces a retraction $h$ of $k[U, V, W, X, Y]$, namely the retraction mapping $X$ and $Y$ to $h^{\prime}(X)$ and $h^{\prime}(Y)$ respectively and leaving $U, V, W$ invariant. This retraction extends in a natural way to retraction of $k\left[U^{ \pm 1}, V^{ \pm 1}, W^{ \pm 1}, X, Y\right]$, and can then be restricted to

$$
k[Q] \otimes k\left[\Delta_{1}\right] \subset k\left[U^{ \pm 1}, V^{ \pm 1}, W^{ \pm 1}, X, Y\right]
$$

where we identify $k[X, Y]$ with the polytopal ring $k\left[\Delta_{1}\right]$ of the unit segment. It can further be restricted to $k\left[\right.$ join $\left.\left(2 Q, 2 \Delta_{1}\right)\right]$ which is embedded into $k[Q] \otimes k\left[\Delta_{1}\right]$ as the tensor product of the second Veronese subalgebras of the normal algebras $k[Q]$ and $k\left[\Delta_{1}\right]$.

We claim that the just constructed retraction $h$ of $k[P], P=\operatorname{join}\left(2 Q, 2 \Delta_{1}\right)$, $\operatorname{dim} P=4$, has no conjugate $h^{\alpha}$ by an automorphism $\alpha \in \Gamma_{k}(P)$ such that the kernel of $h^{\alpha}$ contains a binomial.

The polytope $Q$ has no column structures, a property inherited by $2 Q$. Moreover, both $2 Q$ and $2 \Delta_{1}$ have interior points. Therefore, the only column structures on $P$ are those from $2 \Delta_{1}$ (see Lemma 5.1). Then every element $\alpha \in \Gamma_{k}(P)$ is of the form $\tau \circ \beta$, where $\tau$ is a toric automorphism and $\beta=1 \otimes \beta^{\prime}$ for some $\beta^{\prime} \in \Gamma_{k}\left(2 \Delta_{1}\right)$. Since $\tau$ does not affect the monomial structure, we can assume $\tau=1$. Furthermore, the graded automorphisms of $k\left[2 \Delta_{1}\right]$ are all restrictions of automorphisms of $k\left[\Delta_{1}\right]=$ $k[X, Y]$ so that we have to take into account all automorphisms of $k[P]$ induced by a substitution

$$
X \mapsto a_{11} X+a_{12} Y, \quad Y \mapsto a_{21} X+a_{22} Y, \quad U \mapsto U, \quad V \mapsto V, \quad W \mapsto W
$$

with $\operatorname{det}\left(a_{i j}\right) \neq 0$. Then $h^{\alpha}$ is induced by the substitution

$$
a_{11} X+a_{12} Y \mapsto U+V, \quad a_{21} X+a_{22} Y \mapsto U+W,
$$

leaving $U, V, W$ invariant. Also, $h^{\alpha}$ extends to a retraction of $k\left[U^{ \pm 1}, V^{ \pm 1}, W^{ \pm 1}\right.$, $X, Y]$ and then restricts to $k[U, V, W, X, Y]$. This shows that the kernel of the extension cannot contain a monomial; otherwise, it would contain a monomial in $X$ and $Y$, but $h^{\alpha}$ is injective on $k[X, Y]$. If the kernel contains a binomial $b$, we 
can assume that $b \in k[U, V, W, X, Y]$. In other words, we can find a binomial in the ideal $\mathfrak{p}$ of $k[U, V, W, X, Y]$ generated by

$$
a_{11} X+a_{12} Y-(U+V), \quad a_{21} X+a_{22} Y-(U+W) .
$$

Since the prime ideal $\mathfrak{p}$ contains no monomials, we can assume that the two terms of $b$ are coprime. But then $b$ reduces to a monomial modulo one of the variables, and since $\mathfrak{p}$ reduces to an ideal generated by linear forms, it reduces to a prime ideal. The reduction of $\mathfrak{p}$ modulo any of the variables cannot contain another variable.

In view of what has been said above it is natural to introduce the following classes of tame retractions (for arbitrary codimension) and tame morphisms in $\mathrm{Pol}_{k}$ : a retraction $f: k[P] \rightarrow k[P]$ is called tame if there are a sequence of lattice polytopes $P=P_{m}, P_{m-1} \ldots, P_{1}, P_{0}=Q$, automorphisms $\alpha_{i} \in \Gamma_{k}\left(P_{i}\right)$ and monomial structure preserving retractions $\pi_{i}: k\left[P_{i}\right] \rightarrow k\left[P_{i-1}\right]$ such that

$$
f=\iota \circ \pi_{1} \circ \alpha_{1} \circ \cdots \circ \pi_{m-1} \circ \alpha_{m-1} \circ \pi_{m} \circ \alpha_{m}
$$

for a $k$-algebra embedding $\iota: k[Q] \rightarrow k[P]$.

The definition of a tame morphism is literally the same with the word 'retraction' changed to 'morphism'. Notice that the image of a tame retraction is again a polytopal ring, whereas that of a tame morphism is a semigroup ring of some homogeneous semigroup.

In this terminology Conjecture B merely says that all codimension 1 retractions are tame, while Example 5.2 shows that there are polytopal codimension 2 retractions, which are not even tame morphisms. The following example gives a wild retraction that as a morphism is tame.

Example 5.3. Let $P$ be the join of $7 \Delta_{1}$ and $2 \Delta_{1}$, and thus $\operatorname{dim} P=3$ (see Figure 3). It is straightforward to check that the assignment

$$
B_{1} \mapsto A_{2}+A_{3}, \quad B_{2} \mapsto A_{4}+A_{5}, \quad B_{3} \mapsto A_{6}+A_{7}, \quad A_{i} \mapsto A_{i}, \quad i \in[1,8],
$$

defines a retraction $h$ of $k[P]$, and it is easily checked that $h=\iota \circ g \circ f$, where $\iota$ is the natural embedding of $k\left[7 \Delta_{1}\right]$ into $k[P], f$ is the morphism $k[P] \rightarrow k\left[P^{\prime}\right]$ given by the assignment $A_{i} \mapsto A_{i}^{\prime}, B_{i} \mapsto B_{i}^{\prime}$, and $g: k\left[P^{\prime}\right] \rightarrow k\left[7 \Delta_{1}\right]$ is a tame surjection. Here $P^{\prime}$ is the lattice trapezoid shown in Figure 3. In fact, one can choose $g=\pi_{7 \Delta_{1}} \circ \alpha$, where $\alpha \in \mathbb{A}\left(7 \Delta_{1}\right)$ is an automorphism of $k\left[P^{\prime}\right]$. (The column vectors used for $\alpha$ are indicated in the figure.)

The kernel of $h$ is generated by $B_{1}-\left(A_{2}+A_{3}\right), B_{2}-\left(A_{4}+A_{5}\right), B_{3}-\left(A_{6}+A_{7}\right)$. Assume there is an automorphism $\gamma \in \Gamma_{k}(P)$ such that the image of this ideal contains a binomial or monomial of degree 1 . Then

$$
b=\gamma\left(c_{1}\left(B_{1}-\left(A_{2}+A_{3}\right)\right)+c_{2}\left(B_{2}-\left(A_{4}+A_{5}\right)\right)+c_{3}\left(B_{3}-\left(A_{6}+A_{7}\right)\right)\right)
$$
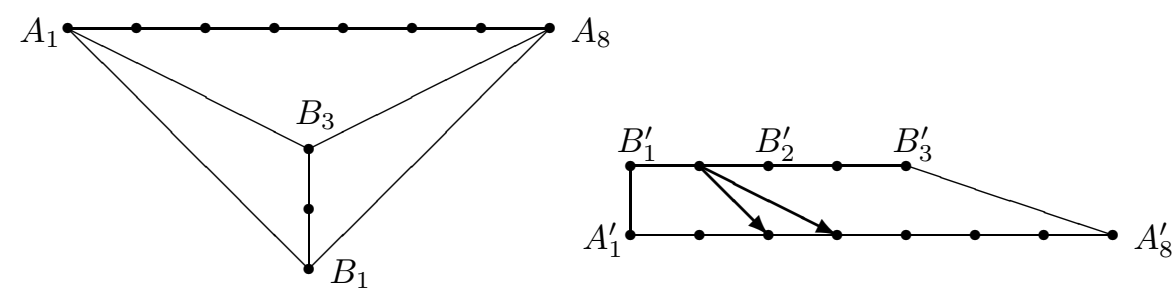

Figure 3 . The polytopes $P$ and $P^{\prime}$ 
is a binomial or monomial for suitable $c_{1}, c_{2}, c_{3}, c_{i} \neq 0$ for at least one $i$. In view of the definition of $P$ as the join of two lattice segments with interior points, we can write $\gamma$ as the composite of two automorphisms induced by automorphisms $\gamma_{1}$ of $k\left[7 \Delta_{1}\right]$ and $\gamma_{2}$ of $k\left[2 \Delta_{1}\right]$, respectively. In particular, if a polynomial contains a term 'on the edge $7 \Delta_{1}$ ', then so does its image under $\gamma$, and the same holds true for the edge $2 \Delta_{1}$. Thus $b$ is not a monomial. Therefore, it cannot be a binomial because then the polynomial

$$
\gamma_{1}\left(c_{1}\left(A_{2}+A_{3}\right)+c_{2}\left(A_{4}+A_{5}\right)+c_{3}\left(A_{6}+A_{7}\right)\right)
$$

would be a term $a$ with

$$
\gamma_{1}^{-1}(a)=c_{1}\left(A_{2}+A_{3}\right)+c_{2}\left(A_{4}+A_{5}\right)+c_{3}\left(A_{6}+A_{7}\right) .
$$

The right-hand side is an 'interior polynomial' of $k\left[7 \Delta_{1}\right]$. On the other hand, an automorphism mapping a term to an 'interior polynomial' must preserve the monomial structure by [BG1, Lemma 4.1].

\section{Segmentonomial ideals}

For an affine semigroup $S$ an element $f \in k[S]$ will be called segmentonomial if the Newton polytope $\mathrm{N}(f) \subset \mathbb{R} \otimes \operatorname{gp}(S)$ has dimension $\leq 1$. (Clearly, monomials as well as binomials are segmentonomials.) An ideal $I \subset k[S]$ is called segmentonomial if it is generated by a system of segmentonomials.

It is proved in [ES] that every minimal prime ideal over a binomial ideal of $k\left[X_{1}, \ldots, X_{n}\right]$ (a polynomial ring) is again binomial. In this section we derive the same result for segmentonomial ideals in arbitrary affine semigroup rings.

Theorem 6.1. Let $S$ be an affine semigroup and $I \subset k[S]$ be a segmentonomial ideal.

(a) A minimal prime overideal $I \subset \mathfrak{p} \subset k[S]$ is binomial, and $k[S] / I$ is again an affine semigroup ring.

(b) Suppose that $\mathrm{ht}(I)=1, f \in I$, $\operatorname{dim}(\mathrm{N}(f))=1$, and $\mathfrak{p}$ is as above. Then for every system of pairwise distinct lattice points $x_{1}, \ldots, x_{m} \in \mathrm{L}_{P}$, such that none of the pairs $\left(x_{i}, x_{j}\right), i \neq j$, spans a line in $\mathbb{R} \otimes \operatorname{gp}(S)$ parallel to $\mathrm{N}(f)$, the residue classes $\bar{x}_{1}, \ldots, \bar{x}_{m}$ constitute a $k$-linearly independent subset of $k[S] / \mathfrak{p}$.

Proof. We prove claim (a) by induction on $r=\operatorname{rank}(S)$. Claim (b) will follow automatically from the description of $\mathfrak{p}$ derived below.

For $r=0$ there is nothing to show. Assume the theorem is proved for semigroups of rank $<r$ and choose a segmentonomial $f \in I$. Then $\mathfrak{p}$ contains a minimal prime $\mathfrak{p}_{0}$ over the principal ideal $(f)$. Assume that $\mathfrak{p}_{0}$ is a binomial ideal. By Theorem [3.4. $k[S] / \mathfrak{p}_{0} \approx k\left[S_{1}\right]$ for some affine semigroup $S_{1}$ and such that monomials in $k[S]$ go to monomials in $k\left[S_{1}\right]$. But then segmentonomials in $k[S]$ are likewise mapped to segmentonomials in $k\left[S_{1}\right]$. This holds true because affinely independent terms lift to affinely independent terms. By induction hypothesis the image of $\mathfrak{p}$ in $k\left[S_{1}\right]$ is binomial. Since binomials can be lifted to binomials in $k[S]$, we conclude that $\mathfrak{p}$ is binomial.

The general situation thus reduces to the case in which $I=(f)$ for some segmentonomial $f \in k[S]$ and $\operatorname{ht}(I)=\operatorname{ht}(\mathfrak{p})=1$.

If $\mathfrak{p}$ contains a monomial, then $\mathfrak{p}$ is a height 1 monomial prime ideal, and we are done. 
Otherwise, $S \cap \mathfrak{p}=\emptyset$. Consider the localization $\mathfrak{p} k[\operatorname{gp}(S)]$. It is a height 1 prime ideal in the Laurent polynomial ring $k[\operatorname{gp}(S)]$. Therefore, $\mathfrak{p} k[\operatorname{gp}(S)]=f_{0} k[\operatorname{gp}(S)]$ for some prime element $f_{0} \in k[\operatorname{gp}(S)]$.

Also $f_{0}$ is segmentonomial. In fact, we have $f=f_{0} f_{1}$ for some $f_{1} \in k[\operatorname{gp}(S)]$ implying the equality $\mathrm{N}(f)=\mathrm{N}\left(f_{0}\right)+\mathrm{N}\left(f_{1}\right)$ for the corresponding Newton polytopes. Since $\operatorname{dim} N\left(f_{0}\right)=0$ is excluded, $\operatorname{dim}\left(\mathrm{N}\left(f_{0}\right)\right)=1$. Multiplying $f_{0}$ by a suitable term from $\operatorname{gp}(S)$ we can achieve that the origin $0 \in \mathbb{R} \otimes \operatorname{gp}(S)$ is one of the end-points of $\mathrm{N}\left(f_{0}\right)$.

Let $\ell \subset \mathbb{R} \otimes \operatorname{gp}(S)$ denote a rational line containing $\mathrm{N}\left(f_{0}\right)$. In a suitable basis of the free abelian group $\operatorname{gp}(S)$ the line $\ell$ becomes a coordinate direction. Therefore, we can assume that

$$
k[\operatorname{gp}(S)]=k\left[X_{1}, X_{1}^{-1}, \ldots, X_{n}, X_{n}^{-1}\right]
$$

and that $f_{0}$ is a monic polynomial in $X_{1}$. Since $k$ is algebraically closed, it follows that $f_{0}=X_{1}-a$ for some $a \in k$. Since $\mathfrak{p}$ does not contain a monomial, one has

$$
\mathfrak{p}=\mathfrak{p} k[\operatorname{gp}(S)] \cap k[S]=\left(X_{1}-a\right) k\left[X_{1}, X_{1}^{-1}, \ldots, X_{n}, X_{n}^{-1}\right] \cap k[S] .
$$

Thus $\mathfrak{p}$ is the kernel of the composite homomorphism

$$
k[S] \hookrightarrow k\left[X_{1}, X_{1}^{-1}, \ldots, X_{n}, X_{n}^{-1}\right] \stackrel{X_{1} \mapsto a}{\longrightarrow} k\left[X_{2}, X_{2}^{-1}, \ldots, X_{n}, X_{n}^{-1}\right] .
$$

This is a homomorphism mapping the elements of $S$ to Laurent monomials in $X_{2}, X_{2}^{-1}, \ldots, X_{n}, X_{n}^{-1}$, and therefore $\mathfrak{p}$ is generated by binomials.

\section{BASED RETRACTIONS}

Throughout this section we suppose that $h: k[P] \rightarrow k[P]$ is a retraction and that $A=\operatorname{Im}(h)$. We also assume $P \subset \mathbb{R}^{n}, \operatorname{dim}(P)=n, \operatorname{gp}\left(S_{P}\right)=\mathbb{Z}^{n+1}$ (and that $k$ is algebraically closed.)

Lemma 7.1. The following conditions are equivalent:

(a) there is a subset $X \subset \mathrm{L}_{P}$ such that the restriction $h: k\left[S_{X}\right] \rightarrow A$ is an isomorphism, where $k\left[S_{X}\right] \subset k[P]$ is the subalgebra generated by the semigroup $S_{X}=\langle X\rangle \subset S_{P}$,

(b) there is a $(\operatorname{dim}(A)-1)$-dimensional cross section $Q$ of $P$ by a linear subspace $H$ such that $Q$ is a lattice polytope (i.e. the vertices of $Q$ are lattice points) and

$$
\left.h\right|_{k[Q]}: k[Q] \rightarrow A
$$

is an isomorphism. In particular, $A$ is a polytopal algebra.

Proof. We only need to derive (b) from (a). Let $H$ be an affine hull of $X$ in $\mathbb{R}^{n}$. We have to show that $Q=H \cap P$ is a lattice polytope with $L_{Q}=X$. Consider the subsemigroup

$$
S_{Q}^{\prime}=\left\{x \in S \mid x \neq 0 \text { and } \mathbb{R}_{+} x \cap P \subset H\right\} \cup\{0\} .
$$

Then $h\left(k\left[S_{Q}^{\prime}\right]\right)=A$ as well. On the other hand,

$$
\operatorname{dim} k\left[S_{Q}^{\prime}\right]=\operatorname{dim} H+1=\operatorname{dim} k\left[S_{X}\right]=\operatorname{dim} A .
$$

Thus the restriction $h: k\left[S_{Q}^{\prime}\right] \rightarrow A$ is also an isomorphism. It follows that $X=L_{Q}$, and every element in $S_{Q}^{\prime}$ is a product of elements of $X$. Furthermore, $Q=\operatorname{conv}(X)$ since any rational point of the complement $Q \backslash \operatorname{conv}(X)$ gives rise to elements in $S_{Q}^{\prime} \backslash S_{X}$ 
A subpolytope $Q \subset P$ as in Lemma 7.1(b) (if it exists) will be called a base of $h$ and $h$ is a based retraction. Notice that a base is not necessarily uniquely determined.

Theorem 7.2. Suppose a retraction $h: k[P] \rightarrow k[P]$ has a base $Q$ that intersects the interior of $P$. Then $h^{\tau}=\iota \circ \rho_{(P, H, W)}$ for some toric automorphism $\tau \in \mathbb{T}_{k}(P)$, a lattice fibration $(P, H, W)$ and a $k$-algebra embedding $\iota: k[H \cap P] \rightarrow k[P]$. In particular, $h$ is tame.

Proof. It is not hard to check that there is no restriction in assuming that $k[Q]=$ $\operatorname{Im}(h)$.

Note that $\operatorname{Ker}(h) \cap S_{P}=\emptyset$. In fact, if a monomial is mapped to 0 by $h$, then $\operatorname{Ker}(h)$ contains a monomial prime ideal $\mathfrak{p}$ of height 1 . Since $\mathfrak{p}$ in turn contains all monomials in the interior of $S_{P}$, it must also contain monomials from $S_{Q}$, which is impossible. Thus $h$ can be extended to the normalization $k\left[\bar{S}_{P}\right]$; on $k\left[\bar{S}_{Q}\right] \subset k\left[\bar{S}_{P}\right]$ the extension is the identity.

Set $L=\mathbb{Z}^{n+1}$, and let $U$ be the intersection of the $\mathbb{Q}$-vector subspace of $\mathbb{Q}^{n+1}$ generated by $S_{Q}$ with $L$. Choose a basis $v_{1}, \ldots, v_{m}$ of a complement of $U$ in $L$. Since $S_{Q}$ contains elements of degree 1 (given by the last coordinate), we can assume that $\operatorname{deg} v_{i}=0$ for $i \in[1, m]$. In sufficiently high degree we can find a lattice point $x$ in $\bar{S}_{Q}$ such that $x v_{i}, x v_{i}^{-1} \in \bar{S}_{P}$. We have the relation $\left(x v_{i}\right)\left(x v_{i}^{-1}\right)=x^{2}$.

It follows that $h\left(x v_{i}\right)=a_{i} x_{i}$, equivalently $h\left(x\left(a_{i}^{-1} v_{i}\right)\right)=x_{i}$, for some $x_{i} \in \bar{S}_{Q}$ and $a_{i} \in k^{*}$. After a toric 'correction' leaving $k\left[\bar{S}_{Q}\right]$ fixed we can assume $a_{i}=1$ for all $i$.

After the inversion of the elements of $S_{P}$, we can further extend the homomorphism $h$ to a map defined on the Laurent polynomial ring $k[L]$. Then we have

$$
h\left(v_{i} x x_{i}^{-1}\right)=1 .
$$

The vectors $v_{i}+x-x_{i}$ are also a basis of a complement of $U$, and thus part of a basis of $L$. Therefore, the elements

$$
v_{i} x x_{i}^{-1}-1, \quad i=[1, m],
$$

generate a prime ideal of height $m$ in $k[L]$.

It is now clear that $h$ (after the toric correction) is just the retraction $\rho_{(P, H, W)}$ where $H$ is the affine hull of $Q$ in $\mathbb{R}^{n}$ and $W$ is the sublattice of $\mathbb{Z}^{n}$ generated by the vectors $v_{i}+x-x_{i}$ upon the identification of $\mathbb{Z}^{n}$ with the degree 0 sublattice of $\mathbb{Z}^{n+1}$.

Example 5.2 shows that even a based retraction $h$ of $k[P]$ need not be tame if the base does not intersect the interior of $P$ and $h$ has codimension $\geq 2$. However, in codimension 1 all based retraction are tame, as follows from Theorem 7.2 and

Theorem 7.3. Suppose the codimension 1 retraction $h: k\left[S_{P}\right] \rightarrow k\left[S_{P}\right]$ has a base $F$ not intersecting the interior of $P$. Then $F$ is a facet of $P$ and $h^{\varepsilon}=\iota \circ \pi_{F}$ for some $\varepsilon \in \mathbb{A}(F)$ and a k-algebra embedding $\iota: k\left[S_{F}\right] \rightarrow k\left[S_{P}\right]$.

In the proof we will use a general fact on pyramids. Recall that a pyramid $\Pi \subset \mathbb{R}^{n}$ is a polytope which is spanned by a point $v$ and a polytope $B$ such that the affine hull of $B$ does not contain $v$. In this situation $v$ is called an apex and $B$ is called a base of $\Pi$. 
Lemma 7.4. Let $\Pi \subset \mathbb{R}^{n}$ be a pyramid and $\Pi=\Pi_{1}+\Pi_{2}$ be a Minkowski sum representation by polytopes $\Pi_{1}, \Pi_{2} \subset \mathbb{R}^{n}$. Then both $\Pi_{1}$ and $\Pi_{2}$ are homothetic images of $\Pi$ (with respect to appropriate centers and non-negative factors).

Proof. The case $\operatorname{dim}(\Pi)=2$ is an easy exercise.

Now we use induction on $\operatorname{dim}(\Pi)$. Assume $\operatorname{dim}(\Pi)=n$ and assume the claim has been shown for pyramids of dimension $\operatorname{dim}(\Pi)-1$. Consider any $(n-1)$-dimensional subspace $\Lambda \subset \mathbb{R}^{n}$ perpendicular to the base $B \subset \Pi$. For a polytope $R \subset \mathbb{R}^{n}$ let $R_{\Lambda}$ denote the image of $R$ in $\Lambda$ under the orthogonal projection $\mathbb{R}^{n} \rightarrow \Lambda$. Then $\Pi_{\Lambda}$ is an $(n-1)$-dimensional pyramid and we have the Minkowski sum representation

$$
\Pi_{\Lambda}=\left(\Pi_{1}\right)_{\Lambda}+\left(\Pi_{2}\right)_{\Lambda}
$$

By induction hypothesis there are homothetic transformations of $\Lambda$ transforming $\Pi_{\Lambda}$ into $\left(\Pi_{1}\right)_{\Lambda}$ and $\left(\Pi_{2}\right)_{\Lambda}$ respectively. Considering all the possible subspaces $\Lambda \subset \mathbb{R}^{n}$ we conclude that

(i) both $\Pi_{1}$ and $\Pi_{2}$ are $n$-pyramids (provided none of them is just a pointin this situation the lemma is obvious) such that the cones they span at corresponding vertices are parallel shifts of the cone spanned by $\Pi$ at its apex $v$

(ii) the corresponding bases of $\Pi_{1}$ and $\Pi_{2}$ are parallel to $B$.

That is exactly what we wanted to show.

Proof of Theorem 7.3. As in the proof of 7.2 we can assume $k[F]=\operatorname{Im}(h)$, and, furthermore, $S_{P} \cap \operatorname{Ker}(h)=\emptyset$, for otherwise $h$ itself passes through a facet retraction. Thus $h$ can be extended to the Laurent polynomial ring $k[L], L=\mathbb{Z}^{n+1}$, and, in particular, to a retraction of $k\left[\bar{S}_{P}\right]$ with image $k\left[\bar{S}_{Q}\right]$. The latter restricts to retractions $k[i P] \rightarrow k[i Q]$ for all $i$. The kernel of the extension $h^{\prime}$ is a height 1 prime ideal and thus principal; $\operatorname{Ker}\left(h^{\prime}\right)=\varphi K[L]$ and $\operatorname{Ker}(h)=(\varphi k[L]) \cap k[P]$ for some element $\varphi \in k[L]$.

Since $F$ is a base of $h, \operatorname{Ker}(h)$ contains the elements $x-\ell, x \in \mathrm{L}_{P} \backslash F, \ell=h(x)$, and $\ell$ is a linear form on the points of $\mathrm{L}_{F}$. Then $\mathrm{N}(\varphi)$ is a Minkowski summand of the pyramid $\mathrm{N}(x-\ell)$ with vertex at $x$. One can shift $\mathrm{N}(\varphi)$ by an integer vector into $\mathrm{N}(x-h(x)) \subset P$ such that the image $R$ satisfies

$$
R \subset P \text { and } R \cap F \neq \emptyset .
$$

Evidently $R$ is the Newton polytope of $y \varphi$ for some $y \in \mathbb{Z}^{n+1}$. Replacing $\varphi$ by $y \varphi$, we can assume that $\mathrm{N}(\varphi)$ satisfies $(* *)$.

By Lemma $7.4 \mathrm{~N}(\varphi)$ is homothetic to $\mathrm{N}(x-\ell)$. Clearly, $F \cap \mathrm{N}(\varphi)$ is a base of $\mathrm{N}(\varphi)$. The corresponding apex of $\mathrm{N}(\varphi)$ is some $z \in \mathrm{L}_{P} \backslash F$.

Consider the valuation

$$
v_{F}: \mathbb{Z}^{n+1} \rightarrow \mathbb{Z}
$$

determined by the conditions:

$$
\operatorname{Im}\left(v_{F}\right)=\mathbb{Z}, v_{F}\left(\mathrm{~L}_{F}\right)=0, v_{F}\left(\mathrm{~L}_{P}\right) \geq 0
$$

We claim that $v_{F}(z)=1$ and $y+b-z \in P$ for any $b \in \mathrm{L}_{F \cap \mathrm{N}(\varphi)}$ and $y \in \mathrm{L}_{P} \backslash F$.

In fact, for $i \in \mathbb{N}$ big enough there is an element $z^{\prime} \in \mathrm{L}_{i P}$ such that $v_{F}\left(z^{\prime}\right)=1$. Since $i F$ is a base of the induced retraction $\bar{h}_{i}: k[i P] \rightarrow k[i P]$, there exists a linear form $\ell^{\prime}$ on $\mathrm{L}_{i F}$ such that $z^{\prime}-\ell^{\prime} \in \operatorname{Ker}(h)$. Thus $\mathrm{N}(\varphi)$ is a Minkowski summand 
of $\mathrm{N}\left(z^{\prime}-\ell^{\prime}\right)$. Because of the condition $(* *)$ we conclude $v_{F}(z) \leq v_{F}\left(z^{\prime}\right)$. Hence $v_{F}(z)=1$.

Now choose $y \in \mathrm{L}_{P} \backslash F$. Since $F$ is a base of $h$, we can write $y-\ell^{\prime \prime} \in \operatorname{Ker}(h)$ for some linear form $\ell^{\prime \prime}$ on the points of $\mathrm{L}_{F}$. Therefore, the pyramid $\mathrm{N}(\varphi)$ is a Minkowski summand of the pyramid $\mathrm{N}\left(y-\ell^{\prime \prime}\right)$ which has its apex at $y$. By Lemma 7.4 the cones spanned by these pyramids at their vertices are the same modulo a parallel shift. This observation in conjunction with the already established equality $v_{F}(z)=1$ makes the claim clear.

We have shown that the vectors $b-z \in \mathbb{Z}^{n}, b \in \mathrm{L}_{F \cap \mathrm{N}(\varphi)}$, are column vectors for $P$. Now, by Lemma 4.1(b) there exists $\varepsilon \in \mathbb{A}(F)$ such that $\varepsilon(\varphi)=c z$ for some $c \in k^{*}$. Therefore, $\operatorname{Ker}\left(h^{\varepsilon}\right)$ is the monomial prime ideal $\left(\mathrm{L}_{P} \backslash F\right) k[P] \subset k[P]$, and this finishes the proof of Theorem 7.3 .

Using Theorem 7.2, Theorem 7.3 and Borel's theorem on maximal tori in linear groups Bor we now derive another sufficient condition for a codimension 1 retraction to be tame.

Theorem 7.5. A codimension 1 retraction $h: k[P] \rightarrow k[P]$ is tame if the graded automorphism group of the $k$-algebra $\operatorname{Im}(h)$ contains a closed $\operatorname{dim}(P)$-torus whose action on $\operatorname{Im}(h)$ extends to an algebraic action on $k[P]$.

Proof. Let $\mathbb{T} \subset$ gr. aut $(\operatorname{Im}(h))$ be such a closed torus. Then there is an algebraic embedding $\mathbb{T} \subset \Gamma_{k}(P)$ and a maximal intermediate torus $\mathbb{T} \subset \mathbb{T}^{\prime} \subset \Gamma_{k}(P)$. By Borel's theorem

$$
\mathbb{T}^{\prime}=\mathbb{T}_{k}(P)^{\alpha}=\left\{\alpha \circ \tau \circ \alpha^{-1} \mid \tau \in \mathbb{T}_{k}(P)\right\}
$$

for some $\alpha \in \Gamma_{k}(P)$. Let us show that $h^{\alpha}$ is a based retraction. By the previous theorems this completes the proof. We can assume that $\operatorname{Ker}\left(h^{\alpha}\right) \subset k[P]$ is not a monomial ideal, or, equivalently, $\operatorname{Ker}\left(h^{\alpha}\right)$ contains no monomials. Otherwise, $h$ factors through a facet projection, and we are done.

Claim. $\operatorname{Ker}\left(h^{\alpha}\right) \subset k[P]$ is a binomial ideal.

We know that $\operatorname{Ker}\left(h^{\alpha}\right)$ is stabilized by the codimension 1 subtorus $\mathbb{T}^{\alpha} \subset \mathbb{T}_{k}(P)$. Since $\operatorname{Ker}\left(h^{\alpha}\right)$ a is non-monomial height 1 prime ideal of $k[P]$, there is an element $\varphi \in k\left[\operatorname{gp}\left(S_{P}\right)\right]$ such that

$$
\operatorname{Ker}\left(h^{\alpha}\right)=k[P] \cap\left(\varphi k\left[\operatorname{gp}\left(S_{P}\right)\right]\right) .
$$

(Compare with the proof of Theorem 6.1) In this situation for any $\tau \in \mathbb{T}^{\alpha}$ we have $\tau(\varphi) k\left[\operatorname{gp}\left(S_{P}\right)\right]=\varphi k\left[\operatorname{gp}\left(S_{P}\right)\right]$. Since $\mathbb{T}^{\alpha}$ is a subtorus of the embedded torus, the latter equation is equivalent to the existence of an element $a_{\tau} \in k^{*}$ such that $\tau(\varphi)=a_{\tau} \varphi . \quad\left(\mathbb{T}^{\alpha}\right.$ acts naturally on coordinate ring $\left.k\left[\mathbb{T}_{k}(P)\right]=k\left[\operatorname{gp}\left(S_{P}\right)\right].\right)$ This means that for any $\tau \in \mathbb{T}^{\alpha}$ the terms in the canonical $k$-linear expansion of $\varphi$ are multiplied by the same scalar from $k^{*}$ when $\tau$ is applied, a condition equivalent to to the segmentonomiality of $\varphi$. In fact, if $\operatorname{dim} N(\varphi) \geq 2$, we would have three non-collinear terms $T_{1}, T_{2}$ and $T_{3}$ in the $k$-linear expansion of $\varphi$, and the condition

$$
\frac{\tau\left(T_{1}\right)}{T_{1}}=\frac{\tau\left(T_{2}\right)}{T_{2}}=\frac{\tau\left(T_{3}\right)}{T_{3}}
$$

on $\tau \in \mathbb{T}_{k}(P)$ would be equivalent to the condition that each $\tau$ is a solution to two independent binomial equations on $\mathbb{T}_{k}(P)=\left(k^{*}\right)^{\operatorname{dim}(P)+1}$. Hence, $\mathbb{T}^{\alpha} \subset \mathbb{T}_{k}(P)$ would be a sub-torus of codimension at least 2 - a contradiction. By this argument 
we have shown that $\varphi$ is a segmentonomial and, hence, a binomial by Theorem 6.1(a). Therefore, by Theorem 3.4 we can assume $\operatorname{Im}(h)=k[S], S=h\left(S_{P}\right)$. In particular,

$$
\sigma_{*}\left(\mathbb{T}^{\alpha}\right)=\mathbb{T}_{k}(S)
$$

where $\sigma_{*}: \operatorname{gr}$. aut $(\operatorname{Im}(h)) \rightarrow \operatorname{gr}$. aut $(k[S])$ is the natural map induced by $\sigma$ and $\mathbb{T}_{k}(S)$ is the embedded torus of $\operatorname{Spec}(k[S])$ corresponding to $S$. The equality holds true because monomial preserving automorphisms are mapped to monomial preserving automorphisms.

Identifying $\operatorname{Im}(h)$ and $k[S]$ via $\sigma$ and $\mathbb{T}^{\alpha}$ and $\mathbb{T}_{k}(S)$ via $\sigma_{*}$ we get a $\mathbb{T}^{\alpha}$-equivariant embedding

$$
\iota: k[S] \rightarrow k[P]
$$

Let $\left\{s_{1}, \ldots, s_{m}\right\}$ be the set of degree 1 terms in $k[S]$. Then for any $\tau \in \mathbb{T}^{\alpha}$ there exist $a_{\tau}^{(i)} \in k^{*}$ such that

$$
\tau\left(\iota\left(s_{i}\right)\right)=a_{\tau}^{(i)} \iota\left(s_{i}\right), \quad i \in[1, m] .
$$

Arguments similar to those in the proof of the claim above show that the polytopes $\mathrm{N}\left(\iota\left(s_{i}\right)\right), i \in[1, m]$, are segments parallel to $\mathrm{N}(\varphi)$, maybe some of them degenerated into points.

Let $z \in \mathbb{R} \otimes \operatorname{gp}\left(S_{P}\right) \backslash\{0\}$ be such that the line $\mathbb{R} z \subset \mathbb{R} \otimes \operatorname{gp}\left(S_{P}\right)$ is parallel to all the $\mathrm{N}\left(\iota\left(s_{i}\right)\right)$. Denote by $t_{i}, i \in[1, m]$ the upper end-points of $\mathrm{N}\left(\iota\left(s_{i}\right)\right)$ in the direction $\mathbb{R}_{+} z$. Then standard arguments with Newton polytopes ensure that the $t_{i}$ are subject to the same affine binomial dependencies as the $s_{i}$. It is now clear that the subpolytope

$$
\operatorname{conv}\left(\left\{t_{1}, \ldots, t_{m}\right\}\right) \subset P
$$

is a base for the retraction $h: k[P] \rightarrow k[S]$.

\section{Retractions of POLygonal AlgEBras}

Throughout this section $P$ denotes a lattice polygon (i.e. $\operatorname{dim}(P)=2$ ).

Theorem 8.1. Any codimension 1 retraction $h: k[P] \rightarrow k[P]$ is based and, therefore, either $h^{\tau}=\iota \circ \rho_{(P, H, w)}$ for some lattice segmental fibrations $(P, H, w), \tau \in$ $\mathbb{T}_{k}(P)$ and a $k$-embedding $\iota: k[H \cap P] \rightarrow k[P]$, or $h^{\varepsilon}=\iota \circ \pi_{F}$ for a facet $F \subset P$, $\varepsilon \in \mathbb{E}_{k}(P)$ and a $k$-embedding $\iota: k[F] \rightarrow k[P]$. In particular, $h$ is tame.

By Theorems 7.2 and 7.3 it is enough to find a base for $h$. The first step in its construction is given by

Proposition 8.2. A multiple $c \Delta_{1}, c \in \mathbb{N}$, of the unit segment $\Delta_{1}$ can be embedded as a lattice polytope into $P$ if and only if there is a $k$-algebra embedding of $k\left[c \Delta_{1}\right]$ into $k[P]$.

Proof. Clearly, without loss of generality, we can assume $c \geq 2$.

Let $\varepsilon: k\left[c \Delta_{1}\right] \rightarrow k[P]$ be an embedding. We write

$$
\mathrm{L}_{c \Delta_{1}}=\left\{x_{0}, x_{1}, \ldots, x_{c}\right\} \text {. }
$$

Thus we have the equations $\varepsilon\left(x_{i-1}\right) \varepsilon\left(x_{i+1}\right)=\varepsilon\left(x_{i}\right)^{2}$ for $i \in[1, c-1]$. Put

$$
\varphi=\frac{\varepsilon\left(x_{1}\right)}{\varepsilon\left(x_{0}\right)}=\frac{\varepsilon\left(x_{2}\right)}{\varepsilon\left(x_{1}\right)}=\cdots .
$$


In the Laurent polynomial ring $k\left[\operatorname{gp}\left(S_{P}\right)\right]=k\left[\mathbb{Z}^{3}\right]$ we can write $\varphi=\varphi_{1} / \varphi_{2}$ with coprime $\varphi_{1}, \varphi_{2}$. The equality $\varphi_{2}^{c} \varepsilon\left(x_{c}\right)=\varphi_{1}^{c} \varepsilon\left(x_{0}\right)$ (and the factoriality of $k\left[\mathbb{Z}^{3}\right]$ ) imply that $\varphi_{1}^{c}$ divides $\varepsilon\left(x_{c}\right)$ and $\varphi_{2}^{c}$ divides $\varepsilon\left(x_{0}\right)$.

Case (a). Both $\varphi_{1}$ and $\varphi_{2}$ are monomials in $k\left[\mathbb{Z}^{3}\right]$. In this situation the Newton polygon $\mathrm{N}\left(\varepsilon\left(x_{c}\right)\right)$ is the parallel shift of $\mathrm{N}\left(\varepsilon\left(x_{0}\right)\right)$ by the $c$ th multiple of the vector representing the support term of the monomial $\varphi$. But then the existence of the desired embedding $c \Delta_{1} \rightarrow \operatorname{conv}\left(\mathrm{N}\left(\varepsilon\left(x_{0}\right)\right), \mathrm{N}\left(\varepsilon\left(x_{c}\right)\right)\right) \subset P$ is obvious.

Case (b). At least one of $\varphi_{1}$ and $\varphi_{2}$, say $\varphi_{1}$, is not a monomial. Then $c \Delta_{1}$ can be embedded in any of the edges of the polygon $\mathrm{N}\left(\varphi_{1}^{c}\right)=c \mathrm{~N}\left(\varphi_{1}\right)$. Since $\varepsilon\left(x_{c}\right)=\psi \varphi_{1}^{c}$ for some $\psi \in k\left[\mathbb{Z}^{3}\right]$, we get

$$
\mathrm{N}\left(\varepsilon\left(x_{c}\right)\right)=\mathrm{N}(\psi)+\mathrm{N}\left(\varphi_{1}^{c}\right)
$$

and the existence of an embedding $c \Delta_{1} \rightarrow \mathrm{N}\left(\varepsilon\left(x_{c}\right)\right) \subset P$ is evident.

Remark 8.3. We expect that Proposition 8.2 holds without any restrictions: for lattice polytopes $P$ and $Q$ a $k$-algebra embedding $k[Q] \rightarrow k[P]$ should only exist if $Q$ can be embedded into $P$ (as a lattice subpolytope).

Below we will need the following notion. For a lattice polygon $P \subset \mathbb{R}^{2}$ its lattice width $\operatorname{width}_{\ell}(P)$ with respect to the line $\ell=z^{\prime}+\mathbb{R} z, z, z^{\prime} \in \mathbb{Z}^{2}, z \neq 0$, is defined as

$$
\operatorname{width}_{\ell}(P)=\max _{x \in P \cap \mathbb{Z}^{2}} \varphi(x)-\min _{y \in P \cap \mathbb{Z}^{2}} \varphi(y)
$$

where $\varphi: \mathbb{Z}^{2} \rightarrow \mathbb{Z}$ is a $\mathbb{Z}$-linear form with $\varphi(z)=0$ and $\varphi(\mathbb{Z})=\mathbb{Z}$. In other words, width $_{\ell}(P)$ is the number of integral translates of $\ell$ intersecting $P$ minus 1 .

Proof of Theorem 8.1. Let $h: k[P] \rightarrow k[P]$ be a codimension 1 retraction. By Theorem 2.2 there is an isomorphism $\operatorname{Im}(h) \approx k\left[c \Delta_{1}\right]$ for some $c \in \mathbb{N}$, and Proposition 8.2 then yields an embedding $c \Delta_{1} \rightarrow P$. Let $Q$ denote its image.

If the restriction of $h$ to the subalgebra $k[Q] \subset k[P]$ is injective, then it is evidently bijective, and we are done by Lemma 7.1. Therefore, we can assume

$$
\operatorname{Ker}(h) \cap k[Q] \neq\{0\} .
$$

Clearly, $I=\operatorname{Ker}(h) \cap k[Q] \subset k[Q]$ is segmentonomial. Since ht $(\operatorname{Ker}(h))=1$ the ideal $\operatorname{Ker}(h)$ is a minimal prime ideal of $I$. If $\operatorname{Ker}(h)$ contains a monomial, then $h$ factors through a facet projection $\pi_{F}$, and then $F$ is a base for $h$. In the other case it is enough to show there is yet another embedding $c \Delta_{1} \rightarrow P$, say with image $R$, such that $Q$ and $R$ are not parallel. Theorem 6.1(b) shows that we then have found a base.

Let $\ell$ denote the line spanned by $Q$. By Theorem 6.1(b) we have

$$
\operatorname{width}_{\ell}(P)=c \text {. }
$$

We use the notation

$$
P_{i}=\mathrm{N}\left(\varepsilon\left(x_{i}\right)\right), \quad i \in[0, c] .
$$

(As in the proof of Proposition 8.2 we write $\mathrm{L}_{c \Delta_{1}}=\left\{x_{0}, x_{1}, \ldots, x_{c}\right\}$.) Evidently, there is no loss in generality in assuming that

$$
P=\operatorname{conv}\left(P_{0} \cup \cdots \cup P_{c}\right)
$$

since, roughly speaking, the retraction can be restricted to the lattice polytope on the right-hand side. 
Case (a). $P_{0} \neq P_{1}$. For every linear form $\lambda: \mathbb{R}^{2} \rightarrow \mathbb{R}$ we consider all triples $\left(\lambda, v_{0}, v_{1}\right)$ such that $v_{0} \in P_{0}, v_{1} \in P_{1}$ and

$$
\lambda\left(v_{i}\right)>\lambda(x) \quad \text { for all } x \in P_{i}, \quad x \neq v_{i}, \quad i=0,1 .
$$

In particular, $v_{0}$ and $v_{1}$ are vertices of $P_{0}$ and $P_{1}$ respectively. We have

$$
i P_{1}=(i-1) P_{0}+P_{i}, \quad i \in[2, c] .
$$

It follows that $\lambda$ has a unique maximum on $P_{i}$, necessarily taken at $v_{i}=i v_{1}-$ $(i-1) v_{0}, i \in[0, c]$. Thus we get the system

$$
\left\{v_{0}, v_{1}, \ldots, v_{c}\right\} \subset P
$$

of collinear lattice points.

Subcase $\left(\mathrm{a}_{1}\right)$. There is a triple $\left(\lambda, v_{0}, v_{1}\right)$ such that $\left[v_{0}, v_{1}\right]$ is not parallel to $\ell$, and in particular, $v_{0} \neq v_{1}$. Then the points $v_{0}, v_{1}, \ldots, v_{c}$ are pairwise different, and so we find $c+1$ successive lattice points in $P$ on a line not parallel to $\ell$.

Subcase $\left(\mathrm{a}_{2}\right)$. In the other case one notes first that the edges of $P_{0}$ and $P_{1}$ are pairwise parallel under an orientation preserving correspondence (in other words, $P_{0}$ and $P_{1}$ have the same normal fans; see [GKZ] for this notion). Moreover, if $v_{0}$ is a vertex of $P_{0}$ and $v_{1}$ is the corresponding vertex of $P_{1}$, then $v_{0}=v_{1}$ or the segment $\left[v_{0}, v_{1}\right]$ is parallel to $\ell$.

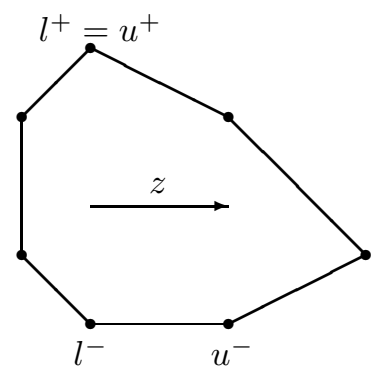

FiguRE 4. Lower and upper boundary

Let us choose $z \in \mathbb{R}^{2}, z \neq 0$ such that $\ell$ is parallel to $\mathbb{R} z$, and let $\xi$ be a non-zero linear form with $\xi(z)=0$.

Furthermore, we consider the upper and lower boundary of $P_{0}$ in direction $z$. (A point $y$ on $\partial P_{0}$ belongs to the lower boundary if the ray in direction $z$ through $y$ enters $P$ at $y$, and the upper boundary is defined analogously; see Figure 4) Each of these subsets has two endpoints $u_{0}^{-}, u_{0}^{+}$and $l_{0}^{-}, l_{0}^{+}$respectively (which may coincide). We choose the indices such that $\xi\left(u_{0}^{-}\right) \leq \xi\left(u_{0}^{+}\right)$and $\xi\left(l_{0}^{-}\right) \leq \xi\left(l_{0}^{+}\right)$. It is clear that $\xi$ takes its minimum on $P_{0}$ in $u_{0}^{-}$and $l_{0}^{-}$and its maximum in the other two points. Applying the same construction to $P_{1}$ we obtain the points $u_{1}^{-}, u_{1}^{+}$and $l_{1}^{-}, l_{1}^{+}$. The correspondence between the vertices of $P_{0}$ and those of $P_{1}$ pairs $u_{0}^{-}$ with $u_{1}^{-}$, etc.

If $u_{0}^{-}=u_{1}^{-}$and $l_{0}^{-}=l_{1}^{-}$, then $P_{0}=P_{1}$ by our initial observation in this subcase. But we are assuming that $P_{0} \neq P_{1}$ in Case (a).

For example, suppose that $u_{0}^{-} \neq u_{1}^{-}$. Then we can find a linear form $\lambda$ such that $u_{0}^{-}$and $u_{1}^{-}$are the unique maxima of $\lambda$ on $P_{0}$ and $P_{1}$ respectively. So the triple $\left(\lambda, u_{0}^{-}, u_{1}^{-}\right)$defines $c+1$ lattice points $v_{0}, \ldots, v_{c}$ in $P$ on a line $\ell^{\prime}$ parallel 
to $\ell$, and one sees easily that $\ell^{\prime}$ intersects $P$ in an edge: it is impossible that $\xi(t)<\xi\left(u_{0}^{-}\right)=\xi\left(u_{1}^{-}\right)$for some $t \in P_{i}, i \in[0, c]$.

Since width $_{\ell}(P)=$ width $_{\ell^{\prime}}(P)=c$, there is a lattice point $t \in P$ such that the triangle $T \subset P$ spanned by $t$ and $c+1$ successive lattice points

$$
w_{0}, w_{1}, \ldots, w_{c} \in \ell^{\prime} \cap \operatorname{conv}\left(\left\{v_{0}, v_{1}, \ldots, v_{c}\right\}\right) \subset P
$$

has $\operatorname{width}_{\ell}(T)=c$. We apply an integral affine transformation $\psi: \mathbb{R}^{2} \rightarrow \mathbb{R}^{2}$ such that $\psi(t)=(0,0)$, and

$$
\psi\left(\operatorname{conv}\left(\left\{w_{0}, v_{1}, \ldots, w_{c}\right\}\right)\right)=\operatorname{conv}((c, r),(c, r+c))
$$

for some $r \in \mathbb{Z}$. There exists $d \in \mathbb{Z}$ such that $d c \in[r, r+c]$. The segment $[(0,0),(c, d c)]$ clearly contains exactly $c+1$ equidistant lattice points and is not parallel to the image of $\ell$ under the transformation $\psi$. Again we are done.

Case (b). $P_{0}=P_{1}$. Then it follows from the equation $i P_{1}=(i-1) P_{0}+P_{i}, i \in[2, c]$ that all the $P_{i}$ coincide and are therefore identical with $P$. If $P$ has an edge containing at least $c+1$ lattice points, then either this edge is not parallel to $Q$ or there is a triangle $T$ as in subcase $\left(\mathrm{a}_{2}\right)$, and in both cases we are done.

Only the case in which every edge of $P$ has lattice length strictly less than $c$ is left. In this situation we resort to the general result below (Proposition 8.4) which we have singled out because of its independent interest.

Proposition 8.4. Assume the edges of the lattice polygon $P \subset \mathbb{R}^{2}$ have lattice lengths strictly less than $c$ and width $_{\ell}(P) \leq c$ for some line $\ell=\mathbb{R} x \subset \mathbb{R}^{2}, x \in \mathbb{Z}^{2}$, $x \neq 0$. Then there is no $k$-algebra embedding $\varepsilon: k\left[c \Delta_{1}\right] \rightarrow k[P]$ such that

$$
P=\mathrm{N}\left(\varepsilon\left(x_{0}\right)\right)=\mathrm{N}\left(\varepsilon\left(x_{1}\right)\right)=\cdots=\mathrm{N}\left(\varepsilon\left(x_{c}\right)\right)
$$

where $x_{0}, \ldots, x_{c}$ denote the successive lattice points of $c \Delta_{1}$.

Proof. Assume to the contrary that such $\varepsilon$ exists. For a subset $W \subset P$ and an element

$$
f=a_{1} y_{1}+\cdots+a_{r} y_{r} \in k[P], \quad a_{j} \in k, \quad y_{j} \in \mathrm{L}_{P},
$$

we put

$$
f_{W}=a_{j_{1}} y_{j_{1}}+\cdots+a_{j_{t}} y_{j_{t}}, \quad\left\{y_{j_{1}}, \ldots, y_{j_{t}}\right\}=W \cap\left\{y_{1}, \ldots, y_{r}\right\} .
$$

Let $E \subset P$ be an edge. It is impossible to find a graded $k$-algebra embedding of $k\left[c \Delta_{1}\right]$ into $k[E]$ (by Hilbert function reasons or Proposition 8.2). In particular, the homomorphism

$$
\pi_{E} \circ \varepsilon: k\left[c \Delta_{1}\right] \rightarrow k[E], \quad x_{i} \mapsto \varepsilon\left(x_{i}\right)_{E},
$$

is not injective. (As usual, $\pi_{E}$ is the facet projection $k[P] \rightarrow k[E]$.) It follows that

$$
\left(\pi_{E} \circ \varepsilon\right)\left(k\left[c \Delta_{1}\right]\right) \approx k\left[\mathbb{Z}_{+}\right] .
$$

as graded $k$-algebras. Thus there is an element $\xi \in k^{*}$ such that

$$
\varepsilon\left(x_{0}\right)_{E}=\xi \varepsilon\left(x_{1}\right)_{E}=\xi^{2} \varepsilon\left(x_{2}\right)_{E}=\cdots=\xi^{c} \varepsilon\left(x_{c}\right)_{E} .
$$

Consider the toric automorphism $\tau: k\left[c \Delta_{1}\right] \rightarrow k\left[c \Delta_{1}\right]$ determined by

$$
x_{i} \mapsto \xi^{i} x_{i}, \quad i \in[0, c] .
$$

Then

$$
\varepsilon^{\prime}\left(x_{0}\right)_{E}=\varepsilon^{\prime}\left(x_{1}\right)_{E}=\cdots=\varepsilon^{\prime}\left(x_{c}\right)_{E},
$$

where $\varepsilon^{\prime}=\varepsilon \circ \tau$. 


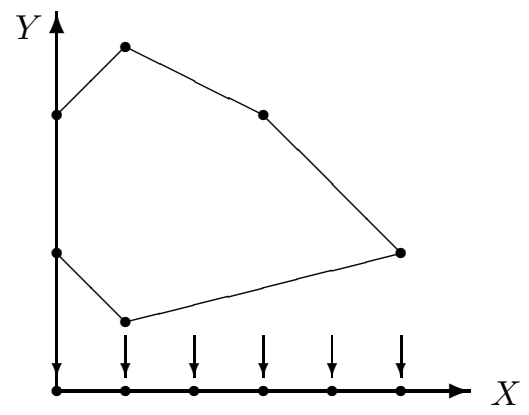

Figure 5. The map $\pi^{a}$

Applying the same arguments to the embedding $\varepsilon^{\prime}: k\left[c \Delta_{1}\right] \rightarrow k[P]$ and an edge $E^{\prime} \subset P$ sharing an end-point with $E$ we conclude

$$
\varepsilon^{\prime}\left(x_{0}\right)_{E^{\prime}}=\varepsilon^{\prime}\left(x_{1}\right)_{E^{\prime}}=\cdots=\varepsilon^{\prime}\left(x_{c}\right)_{E^{\prime}} .
$$

(Because of the common end-point there is no need to further 'correct' $\varepsilon^{\prime}$ by a toric automorphism.) We can assume $\varepsilon=\varepsilon^{\prime}$.

Going once around $P$ we finally arrive at the equations

$$
\varepsilon\left(x_{0}\right)_{\partial P}=\varepsilon\left(x_{1}\right)_{\partial P}=\cdots=\varepsilon\left(x_{c}\right)_{\partial P},
$$

where $\partial P$ denotes the boundary of $P$.

After an integral affine change of coordinates we may assume $\ell=\mathbb{R}(0,1)$ and that $P \subset \mathbb{R}_{+}^{2}$. Then $k[P]$ can be considered as a subalgebra of $k[X, Y, Z]$ generated by monomials $X^{i} Y^{j} Z$. Moreover, after a parallel translation of $P$ we can assume that $P$ contains a lattice point $(0, j)$, or equivalently, $k[P]$ contains a monomial $Y^{j} Z$.

For any element $a \in k^{*}$ we have the retraction

$$
\pi^{a}: k[X, Y, Z] \rightarrow k[X, Z], \quad \pi_{a}\left(X^{i} Y^{j} Z^{m}\right) \mapsto a^{j} X^{i} Z^{m}
$$

for all $i, j, m$. The image of $k[P]$ under $\pi^{a}$ is a polytopal algebra $k\left[c^{\prime} \Delta_{1}\right]$ generated by the elements $Z, X Z, \ldots, X^{c^{\prime}} Z$ with $c^{\prime}=\operatorname{width}_{\ell}(P)$. Due to the infinity of the field $k$ there is $a \in k^{*}$ such that the elements

$$
\pi^{a}\left(\varepsilon\left(x_{i}\right)\right), \quad i \in[0, c],
$$

are pairwise different. In fact, it is clear that for any finite set of polynomials $\mathcal{P} \subset k[X, Y, Z]$ there is an element $a \in k^{*}$ such that $\pi^{a}$ maps $\mathcal{P}$ to a set of $\# \mathcal{P}$ pairwise different polynomials from $k[X, Y]$. (See Figure 5 )

Set

$$
\pi^{a}\left(\varepsilon\left(x_{j}\right)\right)=\sum_{i=0}^{c^{\prime}} \alpha_{i j} X^{i} Z .
$$

In view of the fact that the elements $\varepsilon\left(x_{j}\right)$ coincide 'along $\partial P$ ', we see that the coefficients $\alpha_{0 j}$ coincide for all $j$, and the same holds for the coefficients $\alpha_{c^{\prime} j}$. The $\left(c^{\prime}+1\right) \times(c+1)$ matrix $\left(\alpha_{i j}\right)$ has two linear dependent rows, and since $c^{\prime} \leq c$, its rank is $<c+1$. 
It follows that $\pi^{a} \circ \varepsilon$ is not injective. Thus we see as above that there exists $\eta$ in $k^{*}$ with

$$
\pi^{a}\left(\varepsilon\left(x_{0}\right)\right)=\eta \pi^{a}\left(\varepsilon\left(x_{1}\right)\right)=\eta^{2} \pi^{a}\left(\varepsilon\left(x_{2}\right)\right)=\cdots=\eta^{c} \pi^{a}\left(\varepsilon\left(x_{c}\right)\right) .
$$

Then, however, $\alpha_{00}=\eta \alpha_{01}=\cdots=\eta^{c} \alpha_{0 c}$. By a suitable choice of $a$ we can furthermore achieve that $\alpha_{00} \neq 0$. Then it follows immediately that $\eta=1$. This is a contradiction since we have chosen $a$ such that the $\pi^{a}\left(\varepsilon\left(x_{i}\right)\right)$ are pairwise different.

Remark 8.5. Essentially the same arguments as we have used in $\S 7$ and $\S 8$ imply the analogous results for graded retractions of the semigroup algebras corresponding to affine positive normal semigroups (we only assume all monomials are homogeneous of positive degree) - first, one observes that there is the appropriate notion of a lattice fibration for rational polyhedral pointed cones, and then the arguments are applied to all Veronese subalgebras. (They are essentially the same as polytopal semigroup rings.)

This remark is analogous to Remark 3.3(c) in BG1, saying that the description of graded automorphism groups for graded affine normal semigroup rings is essentially the same as that for polytopal semigroup rings.

\section{REFERENCES}

[Bor] A. Borel, Linear Algebraic Groups, Second edition, Grad. Texts in Math., Springer-Verlag, 1991. MR 92d:20001

[BG1] W. Bruns and J. Gubeladze, Polytopal linear groups, J. Algebra 218 (1999), 715-737. MR 2000g:14059

[BG2] W. Bruns and J. Gubeladze, Polyhedral algebras, toric arrangements, and their groups, Proceedings of the Osaka meeting on computational commutative algebra and combinatorics, 1999. Adv. Stud. Pure Math., to appear.

[BGT] W. Bruns, J. Gubeladze, and N. V. Trung, Normal polytopes, triangulations, and Koszul algebras, J. Reine Angew. Math. 485 (1997), 123-160. MR 96c:52016]

[BH] W. Bruns and J. Herzog. Cohen-Macaulay rings (Rev. Ed.), Cambridge University Press, 1998.

[Cos] D. Costa, Retracts of polynomial rings, J. Algebra 44 (1977), 492-502. MR 55:2876

[ES] D. Eisenbud and B. Sturmfels, Binomial ideals, Duke Math. J. 84 (1996), 1-45. MR 97d:13031

[Fo] R. Fossum, The Divisor Class Group of Krull Domain, Springer-Verlag, 1973. MR 52:3139

[Fu] W. Fulton, Introduction to Toric Varieties, Princeton University Press, 1993. MR 94g:14028

[GKZ] I. M. Gelfand, M. M. Kapranov, and A. V. Zelevinsky, Discriminants, Resultants, and Multidimensional Determinants, Birkhäuser Boston, 1994. MR 95e:14045

[Gi] R. Gilmer, Commutative Semigroup Rings, Univ. Chicago Press, 1984. MR 85e:20058

$[\mathrm{Gu}]$ J. Gubeladze, The isomorphism problem for commutative monoid rings, J. Pure Appl. Algebra 129 (1998), 35-65. MR 99h:20094

[Ha] R. Hartshorne, Algebraic Geometry, Grad. Texts in Math., Springer-Verlag, 1977. MR 57:3116

[Oda] T. Oda, Convex Bodies and Algebraic Geometry (An introduction to the theory of toric varieties), Springer-Verlag, 1988. MR 88m:14038

Universität Osnabrück, FB Mathematik/Informatik, 49069 Osnabrück, Germany

E-mail address: Winfried.Bruns@mathematik.uni-osnabrueck.de

A. Razmadze Mathematical Institute, Alexidze St. 1, 380093 Tbilisi, Georgia

E-mail address: gubel@rmi.acnet.ge 